IMSC $/ 2002 / 07 / 16$

hep-th/0207098

\title{
Loop Variables in String Theory
}

\author{
B. Sathiapalan \\ Institute of Mathematical Sciences \\ Taramani \\ Chennai, India 600113
}

October 24, 2018

\begin{abstract}
The loop variable approach is a proposal for a gauge invariant generalization of the sigma-model renormalization group method of obtaining equations of motion in string theory. The basic guiding principle is space-time gauge invariance rather than world-sheet properties. In essence it is a version of Wilson's exact renormalization group equation for the world sheet theory. It involves all the massive modes and is defined with a finite world-sheet cutoff, which allows one to go off the mass-shell. On shell the tree amplitudes of string theory are reproduced. The equations are gauge invariant off shell also. This article is a self-contained discussion of the loop variable approach as well as its connection with the Wilsonian RG.
\end{abstract}




\section{Introduction}

It has been known for some time now that the renormalization group equations ( $\beta$-functions) for the 2 -dimensional action of a string in a non-trivial background is expected to give the equations of motion for the modes of the string [[1]-[1]]. This is expected to be true both for the closed string modes as well as open string modes. For massless modes, which were the first to be studied, this is relatively easy. In certain limits it can be done to all orders [24, 23. For the tachyon also it has been done in some detail 16] and in some limits can be done to all orders [19, 20, 21, 22].

A natural question to ask is, how can this be extended to the massive modes? There are two complications - one is that when massive modes are involved it is hard to put them all on-shell or even approximately on-shell. Thus one has to learn to deal with off-shell fields. The second complication is that of gauge invariance.

Some of these questions have been addressed in [7, 9, 10, 19, 20]. In [19] an aaproach based on BRST invariance of the world sheet was developed. In this paper, we do not have much to say about this approach. Some of the issues that have to be addressed in this approach are discussed in [20, 21, 22].

For the open string, we argue that the loop variable approach gives an answer to this question. At the free level, equations were written down in [26]. A prescription for the interacting case was given in [27, 28] and many details were worked out in [29, 30, 31].

In [32] a simplification was introduced that made gauge invariance and also other technical issues much more transparent. It is easy to show that the final sytem of equations has the property of being gauge invariant off shell. The relation between these equations and the equations that produce the correct scattering amplitudes for the on-shell physical states, is the same as that between the Wilson renormalization group equations with finite cutoff and the Callan-Symanzik $\beta$-function. Thus one can expect that when one solves for the irrelevant operators one will reproduce the on-shell scattering amplitudes. This method would thus seem (at tree level) to be an alternative to BRST string field theory 37, 36, 38].

This paper explains the results of the loop variable approach in the form given in [32]. It is a self-contained discussion. In order to make it self contained it reviews not only earlier work on loop variables but also contains a short discussion on the Renormalization Group as discussed by Wilson. It then attempts to relate the loop variable approach to the Renormalization Group (RG) and also other standard concepts from field theory. In 
earlier papers on loop variables, many of the constructs were ad-hoc and introduced without any justification other than that they gave the right answer. This paper attempts to motivate some of these ideas by explaining the connection with standard field theoretic constructions. Thus, for instance, the $t$-dependence that was introduced [28] in the loop momenta to make the theory interacting has a very transparent interpretation in terms of "sources" used in defining generating functionals. This paper also attempts to explain in some detail the connection between the loop variable approach and the usual $\beta$-function approach in terms of Wilson's explanation of the RG [13, 12].

This paper is organized as follows. Section 2 discusses some general facts about the connection between Wilson's RG and the Callan-SymanzikGell-Mann-Low $\beta$-functions. It also discusses the role of the irrelevant operators and applies these ideas to the world-sheet RG in string theory. The c-theorem and some applications to cases that can be done in a nonperturbative way (namely, constant e-m field and tachyon with quadratic profile) are also discussed here. Section 3 discusses the same points for the general marginal perturbation, which can only be done perturbatively. Section 4 introduces loop variables as a way of treating all the modes at one go. It also explains the meaning of some of the constructions, by using loop variables to derive some of the results used in section 2 . Section 5 discusses gauge invariance and gives the general solution to the problem posed in the introduction. Section 6 contains some conclusions.

\section{Connection with Wilsonian RG}

\subsection{Generalities about the RG}

The discussion here is based on [12, 13, 14, 15]. Let us begin by assuming that we have a two dimensional action, that corresponds to an open string moving in a completely general open string background. The action has the generic form:

$$
\begin{aligned}
S & =\frac{1}{2} \int_{\Gamma} d^{2} \sigma\left\{\partial^{\alpha} X^{\mu} \partial_{\alpha} X_{\mu}\right\}+\int_{\partial \Gamma} d t L_{1} \\
L_{1} & =\sum_{i} g^{i} M_{i}+\sum_{i} w^{i} W_{i}+\sum_{i} \mu^{i} R_{i}
\end{aligned}
$$

$\mu$ runs from $0-D-1 . D$ is 26 for the bosonic string. $d^{2} \sigma$ is the 
area element in real coordinates and $d t$ the line element. Here $\Gamma$ denotes the (Euclidean) world sheet. Thus at tree level $\Gamma$ is a disc (or upper half plane). $\partial \Gamma$ denotes the boundary of $\Gamma$. Thus $d^{2} \sigma=d x d y$ and $d t=d x$ for the upper half plane.

$L_{1}$ corresponds to the boundary action corresponding to condensation of open string modes. One could also include corrections to the bulk action that correspond to closed string modes in this discussion. Nothing would really be altered. But for concreteness we restrict ourselves to open string backgrounds, which are boundary terms. We denote by $M_{i}, W_{i}$, and $R_{i}$, marginal, irrelevant and relevant operators respectively. $g^{i}, w^{i}, \mu^{i}$, are the corresponding coupling constants. In string theory $g$ would correspond to any mode that is on shell, satisfying $p^{2}=m^{2}$. The off-shell modes would constitute $w, \mu$. At zero momentum, thus, all the massive modes are irrelevant, the photon is marginal, and the tachyon is relevant.

The theory is defined with an ultraviolet cutoff, $\Lambda$. Thus the partition function is

$$
\int_{|p|<\Lambda}[d X(p)] \exp \left\{-S\left[X(p), g_{i}, w_{i}, \mu_{i}\right]\right\}
$$

It is convenient for the purposes of this section to deal with a finite $\mathrm{RG}$ "blocking" transformation that takes the cutoff $\Lambda$ to $\frac{\Lambda}{2}$, rather than making an infinitesimal change. Denote it by $\mathcal{R}$. Thus $\mathcal{R}$ is to be implemented as follows:

1. Perform the integral $\int_{\frac{\Lambda}{2}<|p|<\Lambda}[d X(p)] \exp \{-S[X(p)]\}$.

2. Rescale momenta: Let $p^{\prime}=2 p$. Now the range of $p^{\prime}$ is again $0-\Lambda$.

3. Rescale the surviving $X(p), 0<|p|<\frac{\Lambda}{2}$. Let $X(p)=Z X^{\prime}\left(p^{\prime}\right)$. Choose $Z$ so that the kinetic term $p^{2} X(p) X(-p)$ has the same normalization as before.

As a result of all the above we get an expression for the partition function

$$
\int_{\left|p^{\prime}\right|<\Lambda}\left[d X^{\prime}\left(p^{\prime}\right)\right] \exp \left\{-S\left[X^{\prime}\left(p^{\prime}\right), g_{i}^{\prime}, w_{i}^{\prime}, \mu_{i}^{\prime}\right]\right\}
$$

which is exactly the same as before except that the coupling constants have different values. Thus effectively

$$
\mathcal{R}:(g, w, \mu) \longrightarrow\left(g^{\prime}, w^{\prime}, \mu^{\prime}\right)
$$

defines the discrete renormalization group transformation.

If one plans to iterate the transformation many times one can index it as 


$$
\mathcal{R}:\left(g_{l}, w_{l}, \mu_{l}\right) \longrightarrow\left(g_{l+1}, w_{l+1}, \mu_{l+1}\right)
$$

This defines a recursion relation.

A fixed point would be defined by

$$
\mathcal{R}:\left(g^{*}, w^{*}, \mu^{*}\right) \longrightarrow\left(g^{*}, w^{*}, \mu^{*}\right)
$$

In general there are an infinite number of couping constants, labelled by the superscript $i$ (see (2.1.1)), but for the purposes of this discussion we assume that $i$ takes just one value.

The recursion relation, then, would for instance take the form

$$
\begin{aligned}
\mu_{l+1} & =4 \mu_{l}+N_{\mu}\left[\mu_{l}, g_{l}, w_{l}\right] \\
g_{l+1} & =g_{l}+N_{g}\left[\mu_{l}, g_{l}, w_{l}\right] \\
w_{l+1} & =\frac{1}{4} w_{l}+N_{w}\left[\mu_{l}, g_{l}, w_{l}\right]
\end{aligned}
$$

where the factor 4 characterizes a dimension-2 relevant operator (eg. a mass term, $X^{2}$ ) and the factor $1 / 4$ characterizes a dimension- 4 irrelevant operator, say of the form $(\partial X \partial X)^{2} . N_{\mu}, N_{g}$ and $N_{w}$ correspond to higher order corrections and would have to be small if perturbation theory is to be trusted.

Thus solving for the fixed point would involve setting the coupling constants to satisfy

$$
\begin{aligned}
g_{l} & =g_{l+1} \\
w_{l} & =w_{l+1} \\
\mu_{l} & =\mu_{l+1}
\end{aligned}
$$

Thus if the coupling constants are chosen to be their fixed point values, then doing a block transformation does not change anything. This means that there are efectively no dimensionful physical quantities with which to compare the cutoff $\Lambda$. Thus the theory has an overall scale - the cutoff, $\Lambda$, and no other scale. In particular the correlation lengths must be infinite.

It is important to note that $w^{*} \neq 0$ in general. Thus it is not true in general that the massive modes have zero vev's. Why is it that one usually studies sigma-models with only massless modes turned on? As explained in [13, 12], one can eliminate all the other modes from the equations (2.1.7) for 
the fixed point by solving for them, and the resultant equation involves only the marginal couplings. In order to make this discussion self-contained we summarize the arguments of [13, 12]:

Let $0 \leq l \leq L$ be the range of the index $l$. Thus $\mu_{0}, g_{0}, w_{0}$ are the parameters of the action at high energies. From eqn. (2.1.7) it is clear that to lowest order $\mu_{L} \approx 4^{L} \mu_{0}$. This diverges rapidly with $L$. Thus $\mu_{0}$ has to be tuned very accurately for the long distance effective parameter to have some observed value. This is the famous "fine-tuning" problem. Thus in solving the equations perturbatively we use $\mu_{L}$ as our input. $w_{0}$, on the other hand keeps getting smaller so it can be used as an input parameter. This way it can be seen easily that $\mu_{l}, w_{l}, g_{l}$ rapidly lose their dependence on $w_{0}$ and this is a statement of universality. $g_{l}$ is important for all values of $l$.

Now let us iterate the equations (2.1.7) a number of times. In the case of $\mu_{l}$ the result looks like

$$
\mu_{l}=4^{l-L} \mu_{L}-\sum_{n=l}^{L-1} 4^{l-(n+1)} N_{\mu}\left[\mu_{n}, g_{n}, w_{n}\right]
$$

because we start from $\mu_{L}$ and go backwards. For $w_{l}$ we get

$$
w_{l}=4^{-l} w_{0}+\sum_{n=0}^{l-1} 4^{n+1-l} N_{w}\left[\mu_{n}, g_{n}, w_{n}\right]
$$

For $g_{l}$ we start from $g_{l_{0}}$ and get

$$
g_{l}=g_{l_{0}}+\sum_{n=l_{0}}^{l-1} N_{g}\left[\mu_{n}, g_{n}, w_{n}\right]
$$

We can solve this equation iteratively with the following starting inputs obtained by neglecting the non-linear corrections:

$$
\begin{aligned}
\mu_{l} & =4^{l-L} \mu_{L} \\
w_{l} & =4^{-l} w_{0} \\
g_{l} & =g_{l_{0}}
\end{aligned}
$$

If one solves this set in terms of the input parameters $\mu_{L}, w_{0}, g_{l_{0}}$ one expects a solution of the form

$$
g_{l}=V_{g}\left(g_{l_{0}}, \mu_{L}, w_{0}, l, l_{0}, L\right)
$$


and similarly for $\mu_{l}, w_{l}$.

However if we are in a region where $l>>0$ and also $l<<L$ (which of course means $L>>0$ ) then the equations and solution simplify. Namely the dependence on $\mu_{L}$ and $w_{0}$ of $g_{l}$ is so weak $\left(O\left(4^{l-L}\right.\right.$ and $\left.4^{-l}\right)$ that we can set $\mu_{L}=w_{0}=0$ with negligible error. Furthermore the summations can be extended to $+\infty$ for $\mu_{l}$ and $-\infty$ for $w_{l}$. The resulting equations have a translational invariance in $l$ and $l_{0}$. Thus

$$
g_{l}=V_{g}\left[l-l_{0}, g_{l_{0}}\right]
$$

In physical terms this means that the theory has no dimensionful parameters in this region: Extending the range of $n$ to $-\infty,+\infty$ means the infrared cutoff is zero and UV cutoff is infinity. The only other dimensionful parameters, viz $\mu_{L}$ and $w_{0}$ have been set to zero. The resultant solution for $g_{l}$ can only depend on dimensionless numbers, viz $g_{l_{0}}$ and the ratio of the two scales involved, which is $2^{l-l_{0}}$. In this region the recursion relation can be converted to a differential equation, the usual Gell-Mann - Low, CallanSymanzik $\beta$-function. The solution of this gives us $g^{*}$. One can then solve for $w^{*}$ if one wants.

In string theory for low energy phenomena this calculation thus does not require turning on any massive background fields. If we are interested in low energy phenomena all massive modes are off-shell and do not contribute to the $\beta$-function of the marginal couplings.

What happens if we include a massive mode from the beginning? As long as it scales as an irrelevant operator (which it does at low energies) it is really irrelevant. It doesn't affect the end point of the flow (which is the fixed point). What this means in practice is that in the usual continuum calculation the operator must have the appropriate powers of cutoff in the denominator so that it only renormalizes the marginal coupling and doesn't introduce any new divergences as the cutoff is taken to infinity. However, we repeat: the fixed point value of the irrelevant coupling constant, $w^{*}$, is not zero, and the best way to see this is to look at the exact recursion relations (2.1.7). What can be set to zero is the initial value of the irrelevant coupling $w_{0}$ - as discussed above. Whether $w^{*}$ is of the same order as $g^{*}$ or not depends on the details of the equations.

In string theory, as an example, consider the massive mode $S^{\mu \nu \rho}$ whose equation of motion might typically look like

$$
\left(p^{2}-m^{2}\right) S^{\mu \nu \rho} \approx A^{\mu} A^{\nu} A^{\rho} .
$$


Let us work with dimensionless field variables. Thus we assume that the sigma-model is written in terms of a dimensionless coordinate $X^{\prime \mu}$ where, $X^{\mu}=\sqrt{\alpha^{\prime}} X^{\prime \mu}$. To get fields with canonical dimensions we multiply the field by appropriate powers of $\alpha^{\prime}$. Thus for instance in four dimensions $A^{\prime \mu}=\sqrt{\alpha^{\prime}} A^{\mu}$ and $S^{\prime \mu \nu \rho}=\sqrt{\alpha^{\prime}} S^{\mu \nu \rho}$ are dimensionless fields. The sigmamodel couplings in terms of dimensionless variables are are $\int A^{\prime} \mu \partial_{z} X^{\prime \mu}$ and $\int S^{\prime \mu \nu \rho} \partial_{z} X^{\prime \mu} \partial_{z} X^{\prime} \nu \partial_{z} X^{\prime \rho}$. The equation of motion then is

$$
\alpha^{\prime}\left(p^{2}-m^{2}\right) S^{\prime \mu \nu \rho} \approx A^{\prime \mu} A^{\prime \nu} A^{\prime} \rho
$$

If $A^{\mu}$ has ordinary (weak interaction scale) values then $A^{\prime} \mu \approx \epsilon$ is very small and also $\alpha^{\prime} p^{2} \approx O\left(\epsilon^{2}\right)$. But $\alpha^{\prime} m^{2} \approx 1$. Let us denote $\alpha^{\prime}\left(p^{2}-m^{2}\right)=\delta$. Then $S^{\prime} \approx O\left(\frac{\epsilon^{3}}{\delta}\right)$. So if $\delta \approx O(1)$ then $S^{\prime} \approx O\left(\epsilon^{3}\right)<<A^{\prime}$ On other hand if $\delta \approx O\left(\epsilon^{2}\right)$ then $S^{\prime} \approx O(\epsilon) \approx A^{\prime}$. Similarly if $A^{\prime} \approx O(1)$ then $S^{\prime} \approx O(1)$. Thus the moral of this discussion is that the massive modes can be important for large values of $A$ or for high energies but otherwise they are not significant numerically.

At the fixed point, the physics does not depend on $\Lambda$ so it can be taken to infinity - the continuum limit. In any approximate expression however one can expect explicit powers of the cutoff and it is best to leave $\Lambda$ finite. As an example consider the tachyon vertex operator $\int d x e^{i k X} \Lambda$. The power of $\Lambda$ indicates that it is a relevant operator. If one includes various powers of $\Lambda$ coming from contractions, one gets $: e^{i k X}:\left[1+\frac{k^{2}}{2} \ln \Lambda+\ldots\right] \Lambda$. The expressions $\Lambda \ln \Lambda$ does not have a well defined limit as $\Lambda \rightarrow \infty$, for any value of $k$. However if one sums the entire series, one gets $\Lambda^{\frac{k^{2}}{2}+1}: e^{i k X}:$. For $k^{2}=-2$, it becomes independent of $\Lambda$. Thus the physics becomes independent of the cutoff at the point $k^{2}=-2$, on summing all the loops. But to any finite order one should keep $\Lambda$ finite. This is also the message of the $\epsilon$-expansion 15]. This also illustrates why, in off shell string theory (i.e. when $k^{2} \neq m^{2}$ ), it is important to keep $\Lambda$ finite. This is because different terms in the interacting equation come with different powers of $\Lambda$. It is difficult to make sense of such an equation except when $\Lambda$ is finite. However the series may sum up to some closed form expression in which it may be possible to take $\Lambda$ to $\infty$. Certainly if one has an exact solution to the interacting equation one should find that $\Lambda$-dependence disappears.

Thus the conclusion of this discussion is that while writing down the exact RG equation, one needs to keep a finite cutoff and furthermore the equation will involve all the massive modes. The fixed point will have, in general, non-zero values for the irrelevant couplings, which are the massive 
modes. In the exact expressions for physical correlators, evaluated at the fixed point, the cutoff can be taken to infinity. But in any power series expansion the cutoff must be kept finite. The role of a finite cutoff in string theory RG was illustrated for the tachyon equation in [16].

\subsection{Connection with String Theory}

As discussed above, on-shell string fields correspond to marginal coupling constants for the sigma model and off-shell ones, either to relevant or irrelevant ones. At zero momentum, all the massive fields correspond to irrelevant couplings. The tachyonic fields are relevant couplings. From the discussion of the previous discussion it is thus clear that on-shell string fields behave very differently from off-shell fields.

Sigma model methods have typically been used for marginal fields. This could be near on-shell tachyons, or low momentum vectors. Consider the following action $\left[M_{i}\right.$ are marginal operators]:

$$
S=\frac{1}{2} \int_{\Gamma} d^{2} \sigma\left\{\partial^{\alpha} X^{\mu} \partial_{\alpha} X_{\mu}\right\}+\int_{\partial \Gamma} d t \sum_{i} g^{i} M_{i}(t)
$$

We know that the following relation is true:

$$
\frac{\partial S}{\partial g^{i}}=\beta^{j} G_{i j}
$$

Here $\beta^{i}=\frac{\partial g^{i}}{\partial \ln \Lambda}$ is the $\beta$-function for the coupling constant $g^{i}$ and $G_{i j}$ is the Zamolodchikov metric:

$$
\left\langle M_{i}(z) M_{j}(w)\right\rangle=\frac{G_{i j}}{(z-w)^{2}}
$$

$S$ is an "action" for the couplings. So in string theory this would be the space-time action for the fields of the string. The above is the open string version of the corresponding relation for a general 2-D conformal field theory, which was first shown in [17]. In [16] it was shown to be true for the open string tachyon, to all orders in perturbation theory. This proof followed closely the outline of a proof presented in [18] for closed strings fields in general. The proof can presumably be easily generalized to other operators as well. An outline of a general proof for this is also given in [22].

Using this relation one can proceed as folows. Let $Z\left[g^{i}\right]$ be the partition function corresponding to the action (2.2.15).

$$
Z\left[g^{i}\right]=\int_{|p|<\Lambda}[d X(p)] \exp \left\{-S\left[X(p), g^{i}\right]\right\}
$$


Then

$$
\Lambda \frac{\partial}{\partial \Lambda} \frac{\partial}{\partial g^{i}} Z\left[g^{i}\right]=\beta^{j} \frac{\partial^{2}}{\partial g^{j} \partial g^{i}} Z\left[g^{i}\right]=\int d z \int d w \frac{1}{(z-w)^{2}} \beta^{j} G_{i j}
$$

Thus for marginal operators we get precisely the equation of motion multiplied by an overall factor, the integrals over $(z-w)^{-2}$. More generally, the operator $\Lambda \frac{\partial}{\partial \Lambda}$, produces something proportional to the $\beta$ function, and thus setting $\Lambda \frac{\partial}{\partial \Lambda} Z=\int d w \beta^{i}\left\langle M_{i}(w)\right\rangle=0$ ensures that we are at a fixed point, but it does not produce the full equation of motion.

For off-shell fields we need some generalization. $\left\langle O_{i}(z) O_{j}(w)\right\rangle$ for general (not necessarily marginal) operators $O_{i}$, does not satisfy (2.2.17). One needs a generalization of the Zamolodchikov metric. As an example one can consider

$$
\tilde{G}_{i j}=\frac{1}{L^{2}} \int_{0}^{L} d z \int_{0}^{L} d w(z-w)^{2}\left\langle O_{i}(z) O_{j}(w)\right\rangle
$$

Clearly for (on-shell) exactly marginal fields $\tilde{G}_{i j}=G_{i j}$. Something similar was considered in [19, 20, 21, 22] There the calculation was done on a disc and the prefactor was $\sin ^{2} \frac{\left(\theta_{i}-\theta_{j}\right)}{2}=\left|z_{i}-z_{j}\right|^{2}$, where $z_{i}=e^{i \theta_{i}}, z_{j}=e^{i \theta_{j}}$ are points on the disc. This prescription was derived [19, 20] in a very elegant manner using BRST invariance. However clearly (2.2.19) will not work. One needs to be able to insert a factor of $(z-w)^{2}$ before doing the integral over $z$. In the loop variable approach we will use a different prescription that, as of now, has only been used in a perturbative expansion. This perturbative method is described in Section 3.

We will illustrate some of these ideas with two examples that are exactly computable. One involves marginal operators. This is the case of the uniform electromagnetic field. The other involves relevant operators and is the tachyon with a quadratic profile.

\subsubsection{Born-Infeld}

The ingredients for this calculation are to be found in 23, 24]. $g^{i} O_{i}=$ $\int d k A_{\mu}(k) \partial_{z} X^{\mu} e^{i k . X}$ is added to the boundary. In the limit that $A_{\mu}(k)$ represents a uniform electric/magnetic field, this problem can be done exactly. Thus

$$
\beta^{\nu}=\left(I-F^{2}\right)^{-1 \lambda \mu} \partial_{\lambda} F_{\nu \mu}
$$


is the $\beta$-function to lowest order in derivatives. Similarly

$$
\left\langle\partial_{z} X^{\mu}(z) \partial_{w} X^{\nu}(w)\right\rangle=\frac{1}{(z-w)^{2}} \sqrt{\operatorname{Det}(I+F)}\left(I-F^{2}\right)^{-1 \mu \nu} \equiv \frac{G_{\mu \nu}}{(z-w)^{2}}
$$

is the Zamolodchikow metric [25].

The product gives

$$
\sqrt{\operatorname{Det}(I+F)}\left(1-F^{2}\right)^{-1 \sigma \nu}\left(1-F^{2}\right)^{-1 \lambda \mu} \partial_{\lambda} F_{\nu \mu}=\frac{\delta S}{\delta A_{\sigma}}
$$

where $S=\sqrt{\operatorname{Det}(I+F)}$ is the Born-Infeld action.

The derivation of $(2.2 .21)$ will be done later using loop variable techniques. Here we show how the leading terms arise. This will illustrate the general arguments.

If $Z[A]=\langle 1\rangle_{A}$ is the partition function in the presence of the boundary term, we have

$$
\begin{aligned}
\frac{\partial}{\partial A_{\mu}} Z[A] & =\left\langle\partial_{x} X^{\mu} e^{i k . X}\right\rangle_{A} \\
& =\left\langle\partial_{z} X^{\mu} e^{i k . X}\left\{1+\int d k^{\prime} \int d w \partial_{w} X^{\nu} e^{i k^{\prime} \cdot X} A_{\nu}\left(k^{\prime}\right)+\ldots\right\}\right\rangle
\end{aligned}
$$

The first non zero term is thus

$$
\int d w \int d k^{\prime}\left\langle\partial_{z} X^{\mu} e^{i k \cdot X} \partial_{w} X^{\nu} e^{i k \cdot X} A_{\nu}\left(k^{\prime}\right)\right\rangle
$$

We now have to operate with the RG operator $\Lambda \frac{\partial}{\partial \Lambda}$. The $\Lambda$ dependence comes from coincident two point function which is given by $\left(a=\frac{1}{\Lambda}\right)$,

$$
\lim _{z \rightarrow w}\langle X(z) X(w)\rangle=\lim _{z \rightarrow w} \frac{1}{\pi} \ln (z-w)=\frac{1}{\pi} \ln a=-\frac{1}{\pi} \ln \Lambda
$$

This comes from self contractions in a vertex operator. If we assume that the vector is transverse, then these are of the form

$$
\begin{aligned}
& \int d w \int d k^{\prime}\left\langle\frac{k^{2}}{2} \frac{1}{\pi} \ln \Lambda: \partial_{z} X^{\mu} e^{i k \cdot X}: \partial_{w} X^{\nu} e^{i k^{\prime} \cdot X} A_{\nu}\left(k^{\prime}\right)\right\rangle+ \\
& \int d w \int d k^{\prime}\left\langle: \partial_{z} X^{\mu} e^{i k \cdot X}: \frac{k^{\prime 2}}{2} \frac{1}{\pi} \ln \Lambda \partial_{w} X^{\nu} e^{i k^{\prime} \cdot X} A_{\nu}\left(k^{\prime}\right)\right\rangle \\
& =\left(k^{2}+k^{\prime 2}\right) \frac{\ln \Lambda}{2 \pi} \int d w \int d k^{\prime} \delta\left(k+k^{\prime}\right) \frac{\delta^{\mu \nu} A_{\nu}}{(z-w)^{2}}
\end{aligned}
$$


The RG operator thus gives

$$
\alpha^{\prime} 2 k^{2} A_{\nu}(k)=0
$$

We have used the convention $2 \alpha^{\prime} \pi=1$ This is of course the leading term in the equation of motion for a transverse vector.

We now go to the next order term:

$$
\int d w \int d u \int d v\left\langle\int d k^{\prime} \partial_{z} X^{\mu} e^{i k . X} \frac{F^{\alpha \beta}}{2} X^{\alpha} \partial_{u} X^{\beta}(u) \frac{F^{\gamma \delta}}{2} X^{\gamma} \partial_{v} X^{\delta}(v) \partial_{w} X^{\nu} e^{i k^{\prime} \cdot X} A_{\nu}\left(k^{\prime}\right)\right\rangle
$$

We have brought down three powers of the operator $O_{i}$, and taken the uniform field limit for two of them. By keeping a fixed ordering $z<u<$ $v<w$, we can forget about the factor of $\frac{1}{3 !}$.

Consider the case where the $z, u, v$ operators are first contracted amongst each other. We get $\frac{X^{\rho} X^{\sigma}}{2}$ from $e^{i k . X(z)}$. Since every contraction can be done in two ways, we get a combinatoric factor of 8 that cancels the 8 in the denominator. This gives

$$
\begin{gathered}
\int d w \int d u \int d v \partial_{z} X^{\mu} k^{\rho} k^{\sigma} \delta^{\rho \beta} \partial_{u} G(u-z) \delta^{\alpha \delta} \partial_{v} G(v-u) \delta^{\gamma \sigma} G(v-z) \\
F^{\alpha \beta} F^{\gamma \delta} \partial_{w} X^{\nu} A_{\nu}\left(k^{\prime}\right) \delta\left(k+k^{\prime}\right) \frac{1}{(z-w)^{2}}
\end{gathered}
$$

Here $G(z-w)=\frac{1}{\pi} \ln (z-w)$ and satisfies $\int d u \partial_{u} G(u-z) \partial_{v} G(v-u)=$ $\delta(v-z)$. Thus we get $\int d v G(v-z) \delta(v-z)=G(0)=\frac{1}{\pi} \ln a=-\frac{1}{\pi} \ln \Lambda$. Acting with the RG operator gives $k^{\sigma} F^{\sigma \delta} F^{\delta \rho} k^{\rho} \delta^{\mu \nu} \int d k^{\prime} A_{\nu}\left(k^{\prime}\right) d w \frac{1}{(z-w)^{2}}$. One gets a similar contribution replacing $z \leftrightarrow w$.

Thus the net effect is to replace $k^{2}$ in (2.2.25) by $k^{\rho}\left(I+F^{2}\right)^{\rho \sigma} k^{\sigma}$ which is the expansion of $k^{\rho}\left(I-F^{2}\right)^{-1 \rho \sigma} k^{\sigma}$. The factor of $\sqrt{\operatorname{Det}(I+F)}$ comes from the disconnected vacuum to vacuum amplitude, and multiplies the above expression. In this manner one can build up the full equation of motion.

\subsubsection{Tachyon}

We now consider an off-shell field: A tachyon with profile $\Phi(X)=\frac{T_{0}}{2 \pi}+\frac{1}{2} u X^{2}$. This was worked out in [19, 20, 21, 22]. The world sheet used was a disk.

Using (2.2.23) we see that $\beta_{T_{0}}=-\left(u+T_{0}\right)$ and $\beta_{u}=-u$. Thus to obtain equations of motion we need the generalizations of $G_{u u}, G_{T_{0} T_{0}}, G_{u, T_{0}}$. We

can use for instance the generalized Zamolodchikov metric (2.2.20). The vertex operators are $O_{T_{0}}=1$ and $O_{u}=X^{2}$. 
We will do the calculations on the Upper Half Plane. For a conformally invariant theory this should give the same results as on a disk [19, 20]. However when one is away from the fixed point, i.e. off-shell, one can expect differences from [19, 20]. However, for large $u$, one can expect that the fact that the correlation of $X$ dies out rapidly at long distances would imply insensitivity to the shape of the world sheet. Indeed the partition function calculated on the UHP matches with that on the disk as $u \rightarrow \infty$.

In calculating

$$
G_{T_{0} T_{0}}=\frac{1}{L^{2}} \int_{0}^{L} d z \int_{0}^{L} d w(z-w)^{2}\left\langle\begin{array}{ll}
1 & 1\rangle
\end{array}\right.
$$

and

$$
G_{u T_{0}}=\frac{1}{L^{2}} \int_{0}^{L} d z \int_{0}^{L} d w(z-w)^{2}\left\langle 1 \quad X^{2}(w)\right\rangle
$$

since there is no $z, w$ dependence in the correlation function, the insertion of $(z-w)^{2}$ just gives an overall factor of $\frac{L^{2}}{6}$ compared to the expression without the factor of $(z-w)^{2}$. The expressions without this insertion are given by $\frac{\partial^{2} Z}{\partial_{T_{0}}^{2}}$ and $\frac{\partial^{2} Z}{\partial_{T_{0}} \partial_{u}}$.

$$
G_{u u}=\frac{1}{L^{2}} \int_{0}^{L} d z \int_{0}^{L} d w(z-w)^{2}\left\langle X^{2}(z) \quad X^{2}(w)\right\rangle
$$

is affected because the connected part of the correlation function involves $z-w$.

The leading order term (in powers of $a$ where $a$ is an UV regulator defined in Sec 4.1) in the partition function $Z[u]$, on the UHP is given by (neglecting $T_{0}$ ):

$\ln Z[u]=R \int_{0}^{u} d u^{\prime} e^{u^{\prime} a} E_{i}\left(-u^{\prime} a\right)+b=\frac{R}{a}[-\ln (u a)-C]+R e^{u a} E_{i}(-u a)+b$

$b$ is a constant of integration. This is derived in Sec 4.1. The original derivation in [19] was done on the disc. In the limit $u \rightarrow \infty$ the results are the same.

In the limit $a \rightarrow 0$ we get

$$
\ln Z[u]=R u[\ln (u a)+C]-R u+b \equiv R u(F-1)+b \equiv W+b
$$

Putting back the $T_{0}$ dependence we get

$$
Z[u]=e^{-T_{0}} e^{W+b}
$$




$$
\frac{\partial^{2} Z}{\partial_{T_{0}}^{2}}=Z ; \frac{\partial^{2} Z}{\partial_{T_{0}} \partial_{u}}=-F Z ; \frac{\partial^{2} Z}{\partial_{u}^{2}}=Z\left(\frac{1}{u}+F^{2}\right)
$$

$\frac{Z}{u}$ is the connected part.

Thus $G_{T_{0} T_{0}}=\frac{L^{2}}{6} \frac{\partial^{2} Z}{\partial_{T_{0}}^{2}}=\frac{L^{2}}{6} Z$ and $G_{T_{0} u}=\frac{L^{2}}{6} \frac{\partial^{2} Z}{\partial_{T_{0}} \partial_{u}}=-\frac{L^{2}}{6} F Z$. The

connected part of $G_{u u}$, becomes $\approx \frac{1}{u^{3}} Z$ rather than $\frac{1}{u}$ in $\frac{\partial^{2} Z}{\partial_{u}^{2}}$. Thus in the limit of large $u, u G_{u u}=u Z F^{2}+\frac{Z F^{2}}{u^{2}} \approx u Z F^{2}$.

The equations of motion (in the large $u$ limit) are thus:

$$
\begin{aligned}
\frac{\partial S}{\partial u} & =-\left(F^{2}-F\right) u Z+T_{0} F Z=0 \\
\frac{\partial S}{\partial T_{0}} & =(F-1) u Z-T_{0} Z=0
\end{aligned}
$$

If we plug this in the expression for $Z$, we find that $Z=e^{b}$. By appealing to the small $u$ limit one can fix the normalization of $Z$ (which fixes $b$ ), as in [19, 20]. These equations are also the same as obtained in [19, 20]. One can also see that the two equations are mutually consistent.

Thus we have seen the renormalization group and some of its generalizations being applied in two exactly soluble cases. In the next section we will discuss the general situation where things are not exactly soluble. This will lead us to a different off-shell prescription. One can also try and use the same prescription as [19, 20, 21] for the general case. However it is not obvious how to retain BRST invariance in the presence of a finite cutoff, which is required in the intermediate stages of a calculation of the equations of motion (but which presumably is not required if the fields satisfy those equations).

\section{Equations of Motion for Marginal Perturbations}

\subsection{The General Case}

The Veneziano amplitude for scattering of $\mathrm{N}$ particles has the following form:

$$
\int\left[d z_{3} \ldots . . d z_{N-1}\right]\left[\left\langle V\left(z_{1}\right) V\left(z_{2}\right) V\left(z_{3}\right) \ldots \ldots . . V\left(z_{N-1}\right) V\left(z_{N}\right)\right\rangle\left(z_{1}-z_{2}\right)\left(z_{2}-z_{N}\right)\left(z_{1}-z_{N}\right)\right]
$$

If we set $z_{N}=0$ and take $z_{1} \rightarrow \infty$ this becomes

$$
z_{1}^{2} z_{2} \int\left[d z_{3} \ldots d z_{N-1}\right]\left[\left\langle V\left(z_{1}\right) V\left(z_{2}\right) V\left(z_{3}\right) \ldots \ldots . . V\left(z_{N-1}\right) V(0)\right\rangle\right]
$$


Let us compare this with the following:

$$
z_{1}^{2} \int d z_{2} \int\left[d z_{3} \ldots d z_{N-1}\right]\left[\left\langle V\left(z_{1}\right) V\left(z_{2}\right) V\left(z_{3}\right) \ldots \ldots . . V\left(z_{N-1}\right) V(0)\right\rangle\right]
$$

If one does the usual rescaling $z_{i}^{\prime}=\frac{z_{i}}{z_{2}}$ As $z_{2} \rightarrow 0$, we get a log divergence of the form $\ln \left(z_{1} / a\right)$ where $a \approx \frac{1}{\Lambda}$ is some lattice spacing cutoff. The coefficient of this log divergence is the Veneziano amplitude (3.1.31). This is understood as follows: On shell, the dependence of the correlator on $z_{2}$ is $\frac{1}{z_{2}}$. In (3.1.31), this is cancelled by the factor of $z_{2}$ in front. In (3.1.32), instead of multiplying by $z_{2}$ we integrate the amplitude over $z_{2}$ to get $\int_{a}^{z_{1}} \frac{d z_{2}}{z_{2}}=$ $\ln \left(\frac{z_{1}}{a}\right)$. If we extract the coefficient of this log divergence we get the original amplitude. Similarly, there is a dependence of the form $\left(z_{1}-z_{2}\right)^{-1}$, which produces a $\log$ divergence as $z_{2} \rightarrow z_{1}$, with the same coefficient. All other integrals are also regularized, and it can be shown that this has the effect of subtracting out all intermediate poles [16]. This is the proper time method of extracting the equations of motion for marginal operators (on-shell fields) developed in 16 .

If we considered situations where the operators are not precisely marginal one gets $z_{2}^{-1+\delta}$, with $\delta<<1$ instead of $z_{1}^{-1}$. In this case we get on doing the integral: $\frac{z_{1}^{\delta}-a^{\delta}}{\delta}$. The operation $a \frac{\partial}{\partial a}$ brings a factor of $\delta$, and in the limit of small $\delta$, we have $a^{\delta} \approx 1$. Typically in these calculations $\delta=p^{2}-m^{2}$. However note that for $N=2$ it gives the kinetic term, because there is no integration over $z_{2}$. At higher orders $N \geq 3$, the factors of $p^{2}-m^{2}$ cancel between numerator and denominator, as discussed above, and we are left with the corresponding Veneziano amplitude - minus some of the poles. The poles due to the on-shell particles get subtracted by the regularization of the integrals. Along these lines, in [16] it was shown that one gets the correct equation of motion for the tachyon to all orders in perturbation theory, and also that this equation is proportional to the $\beta$-function, with the proportionality being the Zamolodchikov metric.

\subsection{Tachyon}

Let us illustrate the above ideas with the tachyon [6, 16]. For a tachyon, $V\left(k_{i}, z_{i}\right)=e^{i k_{i} \cdot X\left(z_{i}\right)}$. The perturbation that is added to the sigma-model action is $\int_{\partial \Gamma} d t \int d k \phi(k) e^{i k \cdot X(t)}$. 


\subsubsection{2-point Function}

We consider

$$
\begin{aligned}
& \frac{\partial}{\partial \ln (a)} \int d k_{2}\left\langle\frac{e^{i k_{1} \cdot X\left(z_{1}\right)}}{a} \frac{e^{i k_{2} \cdot X(0)}}{a}\right\rangle \phi\left(k_{2}\right) \\
= & \frac{\partial}{\partial \ln (a)} \int d k_{2} e^{\frac{\left(k_{1}+k_{2}\right)^{2}}{2} \ln a} \frac{e^{k_{1} \cdot k_{2} \frac{1}{\pi} \ln \left(z_{1} / a\right)}}{a^{2}} \phi\left(k_{2}\right) \delta\left(k_{1}+k_{2}\right) \\
= & \frac{\partial}{\partial \ln (a)} e^{\left(\frac{k_{1}^{2}}{\pi}-2\right) \ln a} \phi\left(k_{1}\right) e^{-\frac{k_{1}^{2}}{\pi} \ln z_{1}} \\
\Rightarrow\left(\frac{k_{1}^{2}}{\pi}-2\right) \phi\left(k_{1}\right)= & 2\left(\alpha^{\prime} k_{1}^{2}-1\right) \phi\left(k_{1}\right)=0
\end{aligned}
$$

We have introduced the $a$-dependence due to self-contractions and also used momentum conservation. Since we are using the normalization $2 \pi \alpha^{\prime}=$ 1 , the equation above says that the tachyon mass is $\frac{1}{\alpha^{\prime}}$.

\subsubsection{3-point Function}

This gives a quadratic term in the equation of motion.

We consider

$$
\begin{aligned}
& \frac{\partial}{\partial \ln (a)} \int d k_{2} \int d k_{3} \int_{a}^{z_{1}-a} d z_{2} \lambda\left\langle\frac{e^{i k_{1} \cdot X\left(z_{1}\right)}}{a} \frac{e^{i k_{2} \cdot X\left(z_{2}\right)}}{a} \frac{e^{i k_{3} \cdot X(0)}}{a}\right\rangle \phi\left(k_{2}\right) \phi\left(k_{3}\right) \\
= & \frac{\partial}{\partial \ln (a)} \frac{1}{a^{2}} \int d k_{2} \int d k_{3} \\
& \int_{a}^{z_{1}-a} \frac{d z_{2}}{a}\left(\frac{z_{1}-z_{2}}{a}\right)^{k_{1} \cdot k_{2}}\left(\frac{z_{2}}{a}\right)^{k_{3} \cdot k_{2}}\left(\frac{z_{1}}{a}\right)^{k_{1} \cdot k_{3}} a^{\frac{\left(k_{1}+k_{2}+k_{3}\right)^{2}}{2}} \delta\left(k_{1}+k_{2}+k_{3}\right) \phi\left(k_{2}\right) \phi\left(k_{3}\right) \\
= & \frac{\partial}{\partial \ln (a)} \int d k_{2} a^{-3+k_{1} \cdot k_{3}+k_{2} \cdot k_{3}+k_{2} \cdot k_{3}} \\
& {\left[B\left(k_{1} \cdot k_{2}+1, k_{2} \cdot k_{3}+1\right)-B_{a / z_{1}}\left(k_{1} \cdot k_{2}+1, k_{2} \cdot k_{3}+1\right)-B_{a / z_{1}}\left(k_{2} \cdot k_{3}+1, k_{1} \cdot k_{2}+1\right)\right] } \\
& \times \phi\left(k_{2}\right) \phi\left(-k_{1}-k_{2}\right) \\
= & 2 \lambda \int d k_{2} \phi\left(k_{2}\right) \phi\left(-k_{1}-k_{2}\right)
\end{aligned}
$$

$B_{x}(a, b)$ is the incomplete Beta-function.

$$
B_{x}(a, b)=\int_{0}^{x} t^{a-1}(1-t)^{b-1} d t=\frac{x^{a}}{a}\left[1+\frac{a(1-b)}{a+1} x+\ldots\right]
$$


It is understood that we are taking the limit where the particles are all on shell. In this limit $-3+k_{1} \cdot k_{3}+k_{2} \cdot k_{3}+k_{2} \cdot k_{1} \rightarrow 0$, and there is no $a$-dependence. However when multiplied by the Beta function which has poles, a finite number, namely 2, results for the coefficient of $\ln a$. This implies a $\lambda \frac{\phi^{3}}{3}$ interaction for the tachyon.

\subsubsection{4-point Function}

We consider

$$
\begin{aligned}
& \frac{\partial}{\partial \ln (a)} \int d k_{2} \int d k_{3} \int d k_{4} \int_{a}^{z_{1}-a} d z_{2} \int_{a}^{z_{2}-a} d z_{3} \\
& \lambda^{2}\left\langle\frac{e^{i k_{1} \cdot X\left(z_{1}\right)}}{a} \frac{e^{i k_{2} \cdot X\left(z_{2}\right)}}{a} \frac{e^{i k_{3} \cdot X\left(z_{3}\right)}}{a} \frac{e^{i k_{4} \cdot X(0)}}{a}\right\rangle \phi\left(k_{2}\right) \phi\left(k_{3}\right) \phi\left(k_{4}\right) \\
= & \frac{\partial}{\partial \ln (a)} \frac{1}{a^{2}} \int d k_{2} \int d k_{3} \int d k_{4} \int_{a}^{z_{1}-a} \frac{d z_{2}}{a} \int_{a}^{z_{2}-a} \frac{d z_{3}}{a} \\
& {\left[\left(\frac{z_{1}-z_{2}}{a}\right)^{k_{1} \cdot k_{2}}\left(\frac{z_{1}-z_{3}}{a}\right)^{k_{1} \cdot k_{3}}\left(\frac{z_{2}-z_{3}}{a}\right)^{k_{3} \cdot k_{2}}\left(\frac{z_{1}}{a}\right)^{k_{1} \cdot k_{4}}\left(\frac{z_{2}}{a}\right)^{k_{2} \cdot k_{4}}\left(\frac{z_{3}}{a}\right)^{k_{3} \cdot k_{4}}\right] } \\
= & \frac{\partial}{\partial \ln (a)} \frac{1}{a^{2}} \int d k_{2} \int d k_{3} \int d k_{4}\left(\frac{z_{1}}{a}\right)^{k_{1} \cdot k_{3}+k_{2} \cdot k_{3}+k_{2} \cdot k_{3}+k_{1} \cdot k_{4}+k_{2} \cdot k_{4}+k_{2} \cdot k_{4}+2} \\
& \times \int_{\frac{a}{z_{1}}}^{1-\frac{a}{z_{1}}} d z_{2}^{\prime}\left(1-z_{2}^{\prime}\right)^{k_{1} \cdot k_{2}} z_{2}^{\prime k_{2} \cdot k_{4}+k_{2} \cdot k_{3}+k_{3} \cdot k_{4}+1} a^{\left(k_{1}+k_{2}+k_{3}+k_{4}\right)^{2}} \\
& \times\left[B\left(k_{3} \cdot k_{4}+1, k_{2} \cdot k_{3}+1\right)-B_{a / z_{2}}\left(k_{3} \cdot k_{4}+1, k_{2} \cdot k_{3}+1\right)-B_{a / z_{2}}\left(k_{2} \cdot k_{3}+1, k_{3} \cdot k_{4}+1\right)\right] \\
& \times \phi\left(k_{2}\right) \phi\left(k_{3}\right) \phi\left(k_{4}\right)
\end{aligned}
$$

For convenience we have assumed a configuration where $k_{1} \cdot k_{3} \approx 0$. This factorizes the integral into two parts.

The exponent of $a$ vanishes on shell. Once again the pole terms in the $z_{2}$ integral compensate and we get a factor of 2 mutiplied by:

$\lambda^{2} \int d k_{3} d k_{4}\left[B\left(k_{3} \cdot k_{4}+1, k_{2} \cdot k_{3}+1\right)-\frac{1}{k_{3} \cdot k_{4}+1}-\frac{1}{k_{2} \cdot k_{3}+1}\right] \phi\left(-k_{1}-k_{3}-k_{4}\right) \phi\left(k_{3}\right) \phi\left(k_{4}\right)$.

This is the usual Veneziano four-tachyon amplitude minus the on-shell pole parts.

These calculations illustrate the general idea expressed in Section 3.1. 


\subsection{Vector}

If one restricts oneself to the physical states (transverse) of the vector the calculation simplifies and becomes very similar to that above. We illustrate with the example of the three point fiunction:

$$
\begin{aligned}
& \frac{\partial}{\partial \ln (a)} \frac{1}{a^{2}} \int_{a}^{z_{1}-a} d z_{2} \int d k_{2} \int d k_{3} \\
& \left\langle\partial_{z_{1}} X^{\mu}\left(z_{1}\right) e^{i k_{1} \cdot X\left(z_{1}\right)} \partial_{z_{2}} X^{\nu}\left(z_{2}\right) e^{i k_{2} \cdot X\left(z_{2}\right)} \partial_{z_{3}} X^{\rho}(0) e^{i k_{1} \cdot X(0)}\right\rangle A^{\nu}\left(k_{2}\right) A^{\rho}\left(k_{3}\right) \\
= & \frac{\partial}{\partial \ln (a)} \frac{1}{a^{2}}\left(\frac{z_{1}}{a}\right)^{k_{1} \cdot k_{3}} \int_{a}^{z_{1}-a} \frac{d z_{2}}{a}\left(\frac{z_{1}-z_{2}}{a}\right)^{k_{1} \cdot k_{2}-1}\left(\frac{z_{2}}{a}\right)^{k_{2} \cdot k_{3}-2} \\
= & \frac{\partial}{\partial \ln (a)} \frac{1}{a^{2}} \int d k_{2} \int d k_{3}\left(\frac{z_{1}}{a}\right)^{k_{1} \cdot k_{3}+k_{1} \cdot k_{2}+k_{2} \cdot k_{3}-2} k_{2}^{\mu} A\left(k_{2}\right) \cdot A\left(k_{3}\right) \delta\left(k_{1}+k_{2}+k_{3}\right) \\
& \times\left[B\left(k_{1} \cdot k_{2}, k_{2} \cdot k_{3}-1\right)-B_{a / z_{1}}\left(k_{1} \cdot k_{2}, k_{2} \cdot k_{3}-1\right)-B_{a / z_{1}}\left(k_{2} \cdot k_{3}-1, k_{1} \cdot k_{2}\right)\right]
\end{aligned}
$$

We have assumed transversality of the vector. Furthermore we have picked a particular Wick-contraction that corresponds to a particular term in the equation of motion.

We assume that $k_{1}^{2}=k_{3}^{2}=0$ and $k_{2}^{2}=\epsilon$ to evaluate the expression before taking $\epsilon \rightarrow 0$. We use the expansion (3.2.35). Since we know that the final answer has a log divergence on shell, we keep the coefficient of the log divergence only and throw away everything else and find the same answer, 2. This thus corresponds to the $[A, A][p, A]$ coupling in Yang-Mills theory, for transverse states. It vanishes in electrodynamics for symmetry reasons. If one assumes gauge invariance, one can recover the full coupling from this.

Both, the tachyon and vector calculation described in this section dealt with on-shell physical states. If the procedure is gauge invariant then the rest of the terms in the equation of motion are guaranteed to be correct. The loop variable method (of Sec 5) is one such gauge invariant procedure and gives a gauge covariantized version of the equations derived using the methods of this section. Thus on-shell it is guaranteed to give the right answer. The important thing is that it is gauge invariant off-shell also. Thus it can be used for all the modes at the same time without losing gauge invariance. 


\subsection{Connection with c-theorem}

Before concluding this section we make a connection between the propertime equation of this section and the method given in the last section using Zamolodchikov's c-theorem.

In [16] instead of extracting the $\ln a$ dependence, the $\ln z_{1}$ dependence was extracted. Since $z=e^{\tau}$, this is the "proper time" formalism for strings. It is also shown there that, to all orders, the equation can be written as a product of the $\beta$ - function and the Zamolodchikov meric, as required by the $c$-theorem. One can see the connection as follows:

If $O_{i}$ and $O_{j}$ are almost marginal operators then one has

$$
\left\langle O_{i}(z) O_{j}(0)\right\rangle=\frac{G_{i j}}{z^{2}}+\frac{H_{i j}}{z^{2}} \ln \left(\frac{z}{a}\right)+O\left[\ln ^{2}\left(\frac{z}{a}\right)\right]
$$

where $G_{i j}$ is the Zamolodchikov metric and $H_{i j}$ are the logarithmic deviations from scaling that were calculated in the previous section. If we multiply both sides by $\phi^{j}$ and sum over $j$ we get

$$
\left\langle O_{i}(z) O_{j}(0)\right\rangle \phi^{j}=\frac{G_{i j}}{z^{2}} \phi^{j}+\frac{H_{i j}}{z^{2}} \phi^{j} \ln \left(\frac{z}{a}\right)+O\left[\ln ^{2}\left(\frac{z}{a}\right)\right]
$$

The coefficient of the log in the second term is precisely the proper time equation studied in the last section and is also equal to $G_{i j} \beta^{j}$. Thus we get

$$
\left\langle O_{i}(z) O_{j}(0)\right\rangle \phi^{j}=\frac{G_{i j}}{z^{2}} \phi^{j}+G_{i j} \beta^{j} \ln \left(\frac{z}{a}\right)+O\left[\ln ^{2}\left(\frac{z}{a}\right)\right]
$$

Since $\beta^{j}=-\frac{d \phi^{j}}{d \ln a}$ we see that in the second term $\beta^{j} \ln (z / a) \approx \delta \phi^{j}$. Thus the equation is essentially

$$
\left\langle O_{i}(z) O_{j}(0)\right\rangle \phi^{j}=\frac{G_{i j}}{z^{2}}\left(\phi^{j}+\delta \phi^{j}\right)
$$

Thus we can interpret this equation as follows. If we assume that $z$ is close to $a$ so that we can neglect the higher order terms, then the evolution in $z$ of the state can be seen as a renormalization group evolution of the coupling constants of the corresponding sigma-model.

It is not possible to calculate the unintegrated two-point function from the partition function. It is therefore convenient to calculate the generating functional, which is nothing but the partition function in the presence of a very general background. This leads us to the loop variable of the next section. 


\section{Loop Variables}

The loop variable method is designed to deal with all the modes at one go. However many of the concepts are best illumined by studying some exactly soluble examples, viz. the tachyon with a quadratic profile [19, 20] and also the constant electromagnetic field 23, 24].

\subsection{Tachyon}

We illustrate some of the basic ideas using a simplified example of the tachyon. The best way to deal with very general backgrounds is to use the Fourier transform. Thus define

$$
\phi(X)=\int d k \phi(k) e^{i k \cdot X}
$$

If we solve the problem using $e^{i k . X}$ as the background, then any other background can be treated simply by a suitable integration over the Fourier transform field. A loop variable is an infinite collection of such "vertex operators". Let us illustrate some of the manipulations using this "vertex operator" by applying it to the situation considered in [19, 20] and also in the last section, namely a tachyon with a quadratic profile. We will redo the calculation in the upper half plane as an illustration, thereby also deriving (2.2.26). The main difference between this and the usual vertex operators is that $k$ is not a constant but a current density or a source (See eqn. 4.1.45) below).

$$
S=\frac{1}{2} \int_{\Gamma} d^{2} \sigma\left\{\partial^{\alpha} X^{\mu} \partial_{\alpha} X_{\mu}\right\}-\int_{\partial \Gamma} d t \frac{1}{2} u X_{B}(t)^{2}
$$

Here $X_{B}(t)$ is the value of $X(x, y)$ at the boundary. For the UHP we can write $X_{B}(t)=X(x, 0)$. Defining,

$$
S_{0}=\frac{1}{2} \int_{\Gamma} d^{2} \sigma\left\{\partial^{\alpha} X^{\mu} \partial_{\alpha} X_{\mu}\right\}
$$

we get the partition function correponding to the original action (4.1.43) as follows:

$$
Z[u]=\int \mathcal{D} k \int \mathcal{D} X \exp ^{-S_{0}[X(x, y)]+i \int_{\partial \Gamma} d t k(t) X_{B}(t)} \Psi[k(t), u]
$$

where we have defined a wave-functional,

$$
\Psi[k(t), u]=\operatorname{Det}^{-1 / 2}[2 \pi u] e^{-\frac{1}{2 u} \int_{\partial \Gamma} d t k(t)^{2}} .
$$


We can thus write $Z[u]$ in an obvious way as:

$$
Z[u]=\int \mathcal{D} k W[k(t)] \Psi[k(t), u]
$$

$W[k(t)]$ is a generating functional. In the terminology used in this paper $e^{i \int_{\partial \Gamma} k(t) X(t) d t}$ is the interacting loop variable for the case where there is only one mode - the tachyon.

Thus, once we compute $W[k(t)]$, we can calculate the partition function for any background simply by multiplying by a wave functional appropriate for that background and integrating over $k$. Thus all information about any specific background is contained in $\Psi . W[k]$ needs to be computed only once. Let us do that now.

We write $W$ as

$$
W[k]=\int \mathcal{D} X_{B} \underbrace{\left\{\int \mathcal{D} X e^{-S_{0}} \delta\left[X(u, 0)-X_{B}(u)\right]\right\}}_{F\left[X_{B}\right]} e^{i \int d u k(u) X_{B}(u)}
$$

We are using $u, v: v \geq 0$ as the coordinates of the upper half plane.

In order to evaluate $W$, we write $X(u, v)=X_{c}(u, v)+x(u, v)$ where $X_{c}$ is a solution of the equations of motion satisfying the boundary condition. Thus let $G(z, w)$ be the Green's function that satisfies

$$
\partial \bar{\partial} G(z, w)=\delta^{2}(z-w)
$$

and

$$
\left.G(z, w)\right|_{y=0}=0
$$

We are using the complex notation $z=x+i y, w=u+i v$. In real notation

$$
\partial_{\alpha} \partial^{\alpha} G(x, y ; u, v)=2 \delta(x-u) \delta(y-v)
$$

The solution is

$$
G(z, w)=\frac{1}{\pi}(\ln |z-w|-\ln |z-\bar{w}|)
$$

Then

$$
X_{c}(x, y)=\left.\frac{1}{2} \int d u \partial_{v} G(x, y ; u, v)\right|_{v=0} X_{B}(u)
$$


We can write (after integrating by parts and using properties of the Greens function):

$$
\begin{aligned}
F\left[X_{B}\right] & =e^{\frac{1}{2} \int_{\partial \Gamma} d t X_{c} \partial_{n} X_{c}} \operatorname{Det}^{-1 / 2}\left[\partial^{2}\right] \\
& =e^{-\frac{1}{2 \pi} \int d x \int d u X_{B}(x) \frac{1}{(x-u)^{2}} X_{B}(u)} \operatorname{Det}^{-1 / 2}\left[\partial^{2}\right]
\end{aligned}
$$

If we let

$$
K(x, u)=\frac{1}{\pi} \ln (x-u)
$$

, then

$$
K^{-1}(x, u)=\frac{1}{\pi} \frac{1}{(x-u)^{2}}
$$

$K$ is, in fact, equal to $G_{N}(x, 0 ; u, 0)$ where $G_{N}(x, y: u, v)$ is the Green function satisfying $\partial_{\alpha} \partial^{\alpha} G(x, y ; u, v)=\delta(x-u) \delta(y-v)$, and the Neumann boundary conditions on the real axis : $\left.\partial_{y} G(x, y)\right|_{y=0}=0$.

Thus we get

$$
\begin{aligned}
W[k] & =\int \mathcal{D} X_{B} F\left[X_{B}\right] e^{i \int d x k(x) X_{B}(x)} \\
& =e^{-\frac{1}{2} \int d x \int d u k(x) K(x, u) k(u)} \operatorname{Det}^{1 / 2}[K]
\end{aligned}
$$

Thus, using (4.1.47) and (4.1.46), we can calculate the partition function and other quantities derived from it.

$$
\begin{aligned}
Z[u] & =\int \mathcal{D} k e^{-\frac{1}{2} \int d x \int d u k(x)\left(K(x, u)+\frac{1}{u} \delta(x-u)\right) k(u)} \operatorname{Det}^{1 / 2}[K] \operatorname{Det}^{-1 / 2}[2 \pi u] \\
& =\operatorname{Det}^{-1 / 2}[K+1 / u] \operatorname{Det}^{1 / 2}[K] \operatorname{Det}^{-1 / 2}[u] \\
& =\operatorname{Det}^{-1 / 2}\left[K^{-1}+u\right]
\end{aligned}
$$

where we have dropped $u$-independent constants. In momentum space $K^{-1}(p)=|p|$ and so we get

$$
Z[u]=e^{L \int \frac{d p}{2 \pi} \ln (|p|+u)} .
$$

Here $L$ is the size of the box and is an infrared regulator. If we use a $\mathrm{UV}$ regulator of the form $e^{-a|p|}$ in $K^{-1}$ we get eqn (2.2.26) as the leading approximation.

$\ln Z[u]=R \int_{0}^{u} d u^{\prime} e^{u^{\prime} a} E_{i}\left(-u^{\prime} a\right)+b=\frac{R}{a}[-\ln (u a)-C]+R e^{u a} E_{i}(-u a)+b$ 
$E_{i}(-u a)$ is defined by the equation [43]:

$$
\begin{aligned}
\int_{0}^{\infty} d k \frac{e^{-k a}}{k+u} & =-e^{u a} E_{i}(-u a) \\
& =-e^{u a}\left(C+\ln (u a)+\sum_{n=1}^{\infty} \frac{(-u a)^{n}}{n n !}\right)
\end{aligned}
$$

$C$ is Euler's constant. The above example illustrates some of the basic ideas in using loop variables.

\subsection{Including All Modes}

Loop variables are useful when one has not just one background field as in the above example but an infinite number of them. Thus one is working with the full string field $\Phi[X(s)]$. Thus we have

$$
\Phi[X(s)]=\int[d k(s)] e^{i \int_{c} d s k(s) X(s)} \Phi[k(s)]
$$

But we have to specify how the proper time (or world-sheet time) enters. So we assume the geometry shown in Fig.1.

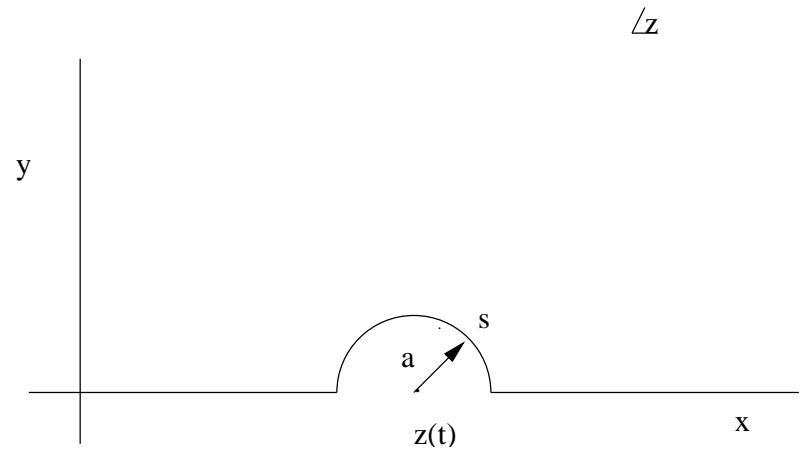

Figure 1: The loop variable is a product over all $t$, of variables defined on a semi-circle of radius $a$ centred around a point $z(t)$ on the edge of the upper half plane.

The location of the vertex operators on the boundary of the world sheet is $z(t)$. At each point we cut out and remove a small semi-circle of radius 
$a$. The boundary of that semi-circle is thus given by $z+a e^{i \theta}=z+a s$. Thus we have $X(z(t)+a s)$, where $z(t)$ is a point on the boundary of the upper half plane, i.e. the real axis. $t$ is a coordinate that parametrizes the real axis. We can in fact choose $z(t)=t$ [31. I The generalized momenta $k(s)$ (or $k_{n}, n \geq 0$ ) also become a generalized current density $k(s, t)$. In the limit $a \rightarrow 0$ we have a collection of vertex operators, all at the same point $z(t)$. We have the following Taylor expansion:

$$
X(z+a s)=X(z)+a s \partial_{z} X(z)+\frac{1}{2 !} a^{2} s^{2} \partial_{z}^{2} X(z)+\ldots
$$

We also assume that $k(s)$ can be expanded in a power seies in $1 / s$. Thus

$$
k(s, t)=k_{0}(t)+\frac{k_{1}(t)}{s}+\frac{k_{2}(t)}{s^{2}}+\ldots
$$

We use the following definition of the loop variable:

$$
e^{\int_{\partial \Gamma} d t i k_{0}(t) X(z(t))+i \int_{\partial \Gamma} d t \int_{c} d s k(s, t) \partial_{z} X(z(t)+a s)}
$$

$\partial \Gamma$ is the real axis when $\Gamma$ is the upper half plane. We will suppress this in subsequent equations. In eqn. (4.2.60) we have separated out the zero mode. In the tachyon case we had only the first term. On performing a Taylor expansion one gets an infinite number of terms from the second term. The loop variable can be written as

$$
e^{i \sum_{n \geq 0} \int d t k_{n}(t) \tilde{Y}_{n}(t)}
$$

where

$$
\tilde{Y}_{n}(t) \equiv \frac{1}{(n-1) !} \partial_{z}^{n} X(z(t)), n>0 ; \quad \tilde{Y}_{0}(t) \equiv X(z(t))
$$

Thus we can define a generating functional $W[k(s, t)]=W\left[k_{0}(t), k_{1}(t), k_{2}(t), \ldots k_{n}(t), \ldots\right]$ (compare (4.1.48) by

$$
\begin{aligned}
W\left[k_{n}(t)\right] & =\left\langle e^{i \int d t \int_{c} k(s, t) \partial_{z} X(z(t)+a s) d s+i \int d t k_{0}(t) X(z(t))}\right\rangle \\
& =\int \mathcal{D} X(u, v) e^{-S_{0}[X(u, v)]} e^{i \int d t \int_{c} k(s, t) \partial_{z} X(z(t)+a s) d s+i \int d t k_{0}(t) X(z(t))} \\
& =\int \mathcal{D} X(u, v) e^{-S_{0}[X(u, v)]} e^{i \sum_{n \geq 0} \int d t k_{n}(t) \tilde{Y}_{n}(t)}
\end{aligned}
$$

\footnotetext{
${ }^{1}$ In some earlier papers [28, 29, 30] $t$ was used as a continuous index labelling the various vertex operators in a correlation function. By setting $z(t)=t$ we are saying that the location itself can be a label and no additional labels are necessary.
} 
Note that $X(z(t))$ and its derivatives are defined on the boundary, whereas the integration is over $X(u, v)$ defined on the upper half plane. Thus $X(z(t))$ is actually $X(z(t), 0)$. We can write (after setting $z(t)=t) X_{B}(t)$ for $X(z(t)$ ) and this can be made explicit by inserting a delta-function and an additional integral over $X_{B}(t)$ as in (4.1.48). Information about a specific background can be encoded in a wave-functional, analogous to (4.1.46), and the partition function can be computed as a function of all the coupling constants, which are nothing but the space-time fields of the open string:

$$
\begin{aligned}
Z\left[\phi^{i}\right] & =\int \mathcal{D} k(s, t) W[k(s, t)] \Psi\left[k(s, t) ; \phi^{i}\right] \\
& =\int \prod_{n \geq 0}\left[\mathcal{D} k_{n}(t)\right] W\left[k_{0}(t), k_{1}(t), . . k_{n}(t), . .\right] \Psi\left[k_{0}(t), k_{1}(t), \ldots k_{n}(t), \ldots ; \phi^{i}\right] .
\end{aligned}
$$

In order to avoid confusion we have made explicit all the $t$-dependences and also the integrations.

\subsection{Uniform Electromagnetic Field}

In Section 4.1 we saw an example of the wave functional in the case where the background was a tachyon with a quadratic profile. Another case that is exactly soluble is the constant electromagnetic field background [23, 24], and will be discussed now as another example. This is discussed in [31]. For simplicity we consider the two dimensional case where we are given a field strength $F^{01}=F$. The partition function can then be written as:

$$
\begin{aligned}
Z[F]= & \iint \mathcal{D} k_{0}^{\mu}(t) \mathcal{D} k_{1}^{\mu}(t) \\
& e^{\frac{1}{2} \iint d t d t^{\prime}\left[\sum_{n, m=0,1} k_{m}^{\mu}(t) \mathcal{G}_{m, n}^{\mu \nu}\left(t, t^{\prime}\right) k_{n}^{\nu}\left(t^{\prime}\right)\right]} \Psi\left[k_{0}(t), k_{1}(t), F^{01}\right] \\
= & \iint \mathcal{D} k_{0}^{\mu}(t) \mathcal{D} k_{1}^{\mu}(t) W\left[k_{n}\right] \Psi\left[k_{0}(t), k_{1}(t), F^{01}\right]
\end{aligned}
$$

Thus we have expressions for both the generating functional $W$ and the partition function $Z$.

Here $\mathcal{G}$ is the matrix

$$
\left(\begin{array}{cccc}
K & 0 & \partial_{t^{\prime}} K & 0 \\
0 & K & 0 & \partial_{t^{\prime}} K \\
\partial_{t} K & 0 & \partial_{t} \partial_{t^{\prime}} K & 0 \\
0 & \partial_{t} K & 0 & \partial_{t} \partial_{t^{\prime}} K
\end{array}\right)
$$


where the row vector, $k_{m}^{\mu}$, ( $\mu$ is the Lorentz index and $m$ is the mode index) multiplying the matrix, is written in the following order: $\left(k_{0}^{0} k_{0}^{1} k_{1}^{0} k_{1}^{1}\right)$. $K\left(t, t^{\prime}\right)=\left\langle X(t) X\left(t^{\prime}\right)\right\rangle$ where $t, t^{\prime}$ are points on the boundary of the string world sheet. This could be a disk or the upper half plane. $K$ was calculated in Section 4.1 to be $\frac{1}{\pi} \ln \left(t-t^{\prime}\right)$ for the upper half plane. $K$ satisfies the identity $\int d t^{\prime \prime} \partial_{t} K\left(t, t^{\prime \prime}\right) \partial_{t^{\prime \prime}} K\left(t^{\prime \prime}, t^{\prime}\right)=1$ [24, 23, 25].

The wave functional in this case is

$$
\Psi\left[k_{0}(t), k_{1}(t) ; F\right]=\int \mathcal{D} X(t) e^{-i \int d t\left[k_{0}^{\mu}(t) X^{\mu}(t)\right]} \prod_{\mu t} \delta\left[k_{1}^{\mu}(t)-A^{\mu}(X(t)]\right.
$$

where $A^{\mu}=1 / 2 F^{\mu \nu} X^{\nu}$, where $\mu=0,1$.

Thus the delta function becomes

$$
\prod_{t} \delta\left[X^{1}(t)-\frac{2 k_{1}^{0}(t)}{F}\right] \delta\left[X^{0}(t)+\frac{2 k_{0}^{1}(t)}{F}\right] \frac{1}{F^{2}}
$$

Doing the the integral gives

$$
\Psi\left[k_{0}(t), k_{1}(t)\right]=e^{\frac{2 i}{F} \int d t\left[k_{0}^{0}(t) k_{1}^{1}(t)-k_{0}^{1}(t) k_{1}^{0}(t)\right]} \prod_{t} \frac{1}{F^{2}}
$$

When we substitute this expression in (4.3.64), we get

$$
\int \mathcal{D} k_{0}(t) \mathcal{D} k_{1}(t) e^{\frac{1}{2} \iint d t d t^{\prime}\left[\sum_{n, m=0,1} k_{m}^{\mu}(t) \mathcal{G}_{F, m, n}^{\mu \nu}\left(t, t^{\prime}\right) k_{n}^{\nu}\left(t^{\prime}\right)\right]}
$$

where $\mathcal{G}_{F}$ is the matrix

$$
\left(\begin{array}{cccc}
K & 0 & \partial_{t^{\prime}} K & \frac{2 i}{F} \\
0 & K & -\frac{2 i}{F} & \partial_{t^{\prime}} K \\
\partial_{t} K & -\frac{2 i}{F} & \partial_{t} \partial_{t^{\prime}} K & 0 \\
\frac{2 i}{F} & \partial_{t} K & 0 & \partial_{t} \partial_{t^{\prime}} K
\end{array}\right)
$$

The Gaussian integral can easily be done and using the identity obeyed by $K$ we get

$$
\begin{gathered}
\operatorname{Det}^{-1 / 2}\left[F^{-2}\left(1+F^{-2}\right) \delta\left(t-t^{\prime}\right)\right]= \\
\operatorname{Det}\left[F^{2}\left(1+F^{2}\right)^{-1 / 2} \delta\left(t-t^{\prime}\right)\right]
\end{gathered}
$$

\footnotetext{
${ }^{2}$ The issue of regularization in defining the wave-functional is discussed in [31]. Since it does not play a significant role in this calculation we do not discuss it here.
} 
Combining the $\frac{1}{F^{2}}$ in the wave functional we get

$$
\operatorname{Det}\left[1+F^{2}\right]^{-1 / 2} \delta\left(t-t^{\prime}\right)
$$

Using zeta function regularization (as explained in 23]) the determinant gives $\left[1+F^{2}\right]^{+1 / 2}$. Thus the final answer is

$$
Z[F]=\left[1+F^{2}\right]^{+1 / 2} .
$$

In order to obtain the equation of motion we can follow the prescription of (2.2.19) reproduced here:

$$
\Lambda \frac{\partial}{\partial \Lambda} \frac{\partial}{\partial g^{i}} Z\left[g^{i}\right]=\beta^{j} \frac{\partial^{2}}{\partial g^{j} \partial g^{i}} Z\left[g^{i}\right]=\int d z \int d w \frac{1}{(z-w)^{2}} \beta^{j} G_{i j}
$$

We thus need to calculate

$$
\begin{aligned}
\Lambda \frac{\partial}{\partial \Lambda}\left\langle O_{i}\right\rangle & =\beta^{j} \int d t^{\prime}\left\langle O_{j}\left(t^{\prime}\right) O_{i}(t)\right\rangle \\
& =\beta^{j} \int d t^{\prime} \frac{1}{\left(t-t^{\prime}\right)^{2}} G_{j i}
\end{aligned}
$$

The operator $O_{i}$ in our case is $\dot{X}^{\mu}$. We can thus calculate

$$
\begin{aligned}
\left.\left\langle\dot{X}^{\mu}\left(t^{\prime \prime}\right)\right\rangle\right|_{A}= & \iint \mathcal{D} k_{0}^{\mu}(t) \mathcal{D} k_{1}^{\mu}(t) \\
& \frac{\delta}{\delta k_{1}^{\mu}\left(t^{\prime \prime}\right)}\left[e^{\frac{1}{2} \iint d t d t^{\prime}\left[\sum_{n, m=0,1} k_{m}^{\mu}(t) \mathcal{G}_{m, n}^{\mu \nu}\left(t, t^{\prime}\right) k_{n}^{\nu}\left(t^{\prime}\right)\right]}\right] \\
& \times \Psi\left[k_{0}(t), k_{1}(t), F\right] \\
= & \iint \mathcal{D} k_{0}^{\mu}(t) \mathcal{D} k_{1}^{\mu}(t) \frac{\delta}{\delta k_{1}^{\mu}\left(t^{\prime \prime}\right)} W\left[k_{n}\right] \Psi\left[k_{0}(t), k_{1}(t), F^{01}\right]
\end{aligned}
$$

It is quite clear from the symmetry of $W$ and $\Psi$ (both are even in $k$ ) that this is zero. Thus the equation of motion is trivial. When the electromagnetic field is uniform the theory is conformal. We therefore need to consider next order corrections due to non-uniformity of the electromagnetic field. We thus take

$$
A^{\mu}(X)=\frac{1}{2} F^{\mu \nu} X^{\nu}+\frac{1}{3} \partial_{\rho} F^{\mu \nu} X^{\rho} X^{\nu}
$$


The delta function in 4.3 .66 changes to

$$
\begin{gathered}
\prod_{t} \delta\left[k_{1}^{\mu}(t)-\frac{1}{2} F^{\mu \nu} X^{\nu}(t)+\frac{1}{3} \partial_{\rho} F^{\mu \nu} X^{\rho}(t) X^{\nu}(t)\right] \\
=\left(1+\int d t^{\prime} \frac{1}{3} \partial_{\rho} F^{\mu \nu} X^{\rho}\left(t^{\prime}\right) X^{\nu}\left(t^{\prime}\right) \frac{\delta}{\delta k_{1}^{\alpha}\left(t^{\prime}\right)}\right) \prod_{t} \delta\left[k_{1}^{\mu}(t)-\frac{1}{2} F^{\mu \nu} X^{\nu}(t)\right]
\end{gathered}
$$

If we insert this into (4.3.66) we get for the modified wave functional,

$$
\begin{gathered}
\Psi\left[k_{0}(t), k_{1}(t) ; F\right]=\int \mathcal{D} X(t) e^{-i \int d t\left[k_{0}^{\mu}(t) X^{\mu}(t)\right]} \\
\left(1+\int d t^{\prime} \frac{1}{3} \partial_{\rho} F^{\mu \nu} X^{\rho}\left(t^{\prime}\right) X^{\nu}\left(t^{\prime}\right) \frac{\delta}{\delta k_{1}^{\alpha}\left(t^{\prime}\right)}\right) \prod_{\mu t} \delta\left[k_{1}^{\mu}(t)-A^{\mu}(X(t)]\right. \\
=\left(1+\int d t^{\prime} \frac{1}{3} \partial_{\rho} F^{\mu \nu} i \frac{\delta}{\delta k_{0}^{\rho}\left(t^{\prime}\right)} i \frac{\delta}{\delta k_{0}^{\nu}\left(t^{\prime}\right)} \frac{\delta}{\delta k_{1}^{\alpha}\left(t^{\prime}\right)}\right) \Psi\left[k_{0}(t), k_{1}(t) ; F\right]
\end{gathered}
$$

We insert this into 4.3.72 and integrate by parts the derivatives on $\Psi$, to get

$$
\begin{gathered}
\left\langle\dot{X}^{\alpha}\right\rangle \approx \int d t^{\prime}\left\{\left[\mathcal{G}-\mathcal{G} \mathcal{G}_{F}^{-1} \mathcal{G}\right]_{0 \nu, 1 \mu}\left(t^{\prime}, t^{\prime}\right)\left[\mathcal{G}-\mathcal{G} \mathcal{G}_{F}^{-1} \mathcal{G}\right]_{0 \rho, 1 \alpha}\left(t^{\prime}, t^{\prime \prime}\right)+\right. \\
{\left[\mathcal{G}-\mathcal{G} \mathcal{G}_{F}^{-1} \mathcal{G}\right]_{0 \rho, 1 \mu}\left(t^{\prime}, t^{\prime}\right)\left[\mathcal{G}-\mathcal{G} \mathcal{G}_{F}^{-1} \mathcal{G}\right]_{0 \nu, 1 \alpha}\left(t^{\prime}, t^{\prime \prime}\right)} \\
\left.+\left[\mathcal{G}-\mathcal{G} \mathcal{G}_{F}^{-1} \mathcal{G}\right]_{0 \nu, 0 \rho}\left(t^{\prime}, t^{\prime}\right)\left[\mathcal{G}-\mathcal{G} \mathcal{G}_{F}^{-1} \mathcal{G}\right]_{1 \alpha, 1 \mu}\left(t^{\prime}, t^{\prime \prime}\right)\right\} \partial_{\rho} F_{\mu \nu} \\
=\left\{\mathcal{K}_{0 \nu, 1 \mu}\left(t^{\prime}, t^{\prime}\right) \mathcal{K}_{0 \rho, 1 \alpha}\left(t^{\prime}, t^{\prime \prime}\right)+\mathcal{K}_{0 \rho, 1 \mu}\left(t^{\prime}, t^{\prime}\right) \mathcal{K}_{0 \nu, 1 \alpha}\left(t^{\prime}, t^{\prime \prime}\right)+\mathcal{K}_{0 \nu, 0 \rho}\left(t^{\prime}, t^{\prime}\right) \mathcal{K}_{1 \alpha, 1 \mu}\left(t^{\prime}, t^{\prime \prime}\right)\right\} \partial_{\rho} F_{\mu \nu}
\end{gathered}
$$

(This equation defines $\mathcal{K}$ in an obvious way.)

Using the expressions (4.3.65), 4.3.68) for $\mathcal{G}$ and $\mathcal{G}_{F}$ one finds:

We are using the following notation: Products of $K$ are understood as convolution. Thus $K K=\int d t^{\prime} K\left(t, t^{\prime}\right) K\left(t^{\prime}, t^{\prime \prime}\right)$. Also $\partial_{t^{\prime}} K=\partial_{t^{\prime}} K\left(t, t^{\prime}\right)$ and $\ddot{K}=\partial_{t} \partial_{t^{\prime}} K\left(t, t^{\prime}\right)$.

$$
\left[\mathcal{G}-\mathcal{G} \mathcal{G}_{F}^{-1} \mathcal{G}\right]_{1 \mu, 1 \nu}\left(t, t^{\prime}\right)=\mathcal{K}_{1 \mu, 1 \nu}\left(t, t^{\prime}\right)=\left(\begin{array}{cc}
\frac{\ddot{K}}{1+F^{2}} & -i \frac{F \partial_{\tau^{\star}} \delta\left(\tau-\tau^{\prime \prime}\right)}{1+F^{2}} \\
i \frac{F \partial_{\tau^{\prime}} \delta\left(\tau-\tau^{\prime \prime}\right)}{1+F^{2}} & \frac{\ddot{K}}{1+F^{2}}
\end{array}\right)
$$




$$
\begin{aligned}
& {\left[\mathcal{G}-\mathcal{G} \mathcal{G}_{F}^{-1} \mathcal{G}\right]_{0 \mu, 1 \nu}\left(t, t^{\prime}\right)=\mathcal{K}_{0 \mu, 1 \nu}\left(t, t^{\prime}\right)=\left(\begin{array}{cc}
\frac{\partial_{\tau^{\prime}} K}{1+F^{2}} & 0 \\
0 & \frac{\partial_{\tau^{\prime}} K}{1+F^{2}}
\end{array}\right)} \\
& {\left[\mathcal{G}-\mathcal{G} \mathcal{G}_{F}^{-1} \mathcal{G}\right]_{0 \mu, 0 \nu}\left(t, t^{\prime}\right)=\mathcal{K}_{0 \mu, 0 \nu}\left(t, t^{\prime}\right)=\left(\begin{array}{cc}
\frac{K}{1+F^{2}} & -\frac{i F K \partial_{\tau^{\prime}} K}{1+F^{2}} \\
\frac{i F K \partial_{\tau^{\prime}} K}{1+F^{2}} & \frac{K}{1+F^{2}}
\end{array}\right)}
\end{aligned}
$$

Using these expressions for $\mathcal{K}$ in 4.3 .75 ) one finds (using $K\left(t^{\prime}-t^{\prime}\right)=\ln a$, and $\partial_{t} K\left(t-t^{\prime}\right)=0$ when $t \rightarrow t^{\prime}$ by antisymmetry),

$$
\left.\left\langle\dot{X}^{\alpha}\left(t^{\prime \prime}\right)\right\rangle\right|_{A}=\ln a \int d t^{\prime} \delta_{\rho \nu} \delta_{\mu \alpha}\left(\frac{1}{1+F^{2}}\right)^{2} \frac{1}{\left(t^{\prime}-t^{\prime \prime}\right)^{2}} \times \partial_{\rho} F_{\mu \nu} Z[F]
$$

Thus setting $\frac{d}{d \ln a}\left\langle\dot{X}^{\alpha}\left(t^{\prime \prime}\right)\right\rangle=0$ gives the Born-Infeld equation of motion. Note that $Z[F]$ is regularized. This is because we need only the term linear in $\ln a$ and we already have one power of $\ln a$.

\subsection{General Case: Perturbation Theory}

We have seen how loop variables can be used in two special cases where the problem is exactly soluble. For general backgrounds we cannot calculate $Z\left[g^{i}\right]$ exactly. Nevertheless $W[k(s, t)]=W\left[k_{n}(t)\right]$ is always the same and can be calculated very easily.

It is in fact given by eqn (4.4.80):

$$
\begin{aligned}
W\left[k_{n}(t)\right] & =\left\langle e^{i \int d t \int_{c} k(s, t) \partial_{z} X(z(t)+a s) d s+i \int d t k_{0}(t) X(z(t))}\right\rangle \\
& =\int \mathcal{D} X e^{-S_{0}[X]} e^{i \int d t \int_{c} k(s, t) \partial_{z} X(z(t)+a s) d s+i \int d t k_{0}(t) X(z(t))} \\
& =\int \mathcal{D} X e^{-S_{0}[X]} e^{i \sum_{n \geq 0} \int d t k_{n}(t) \tilde{Y}_{n}(t)}
\end{aligned}
$$

Using the result that $\left\langle X(t) X\left(t^{\prime}\right)\right\rangle=K\left(t, t^{\prime}\right)$ (see (4.1.53)), we can write this as

$$
W\left[k_{n}(t)\right]=e^{-\frac{1}{2} \int d t \int d t^{\prime} \sum_{n, m \geq 0} k_{n}(t) K_{n, m}\left(t, t^{\prime}\right) k_{m}\left(t^{\prime}\right)}
$$

where

$$
\begin{aligned}
K_{n, m} & =\left\langle\tilde{Y}_{n}(t) \tilde{Y}_{m}\left(t^{\prime}\right)\right\rangle \\
& =\frac{1}{(n-1) !} \frac{1}{(m-1) !} \partial_{t}^{n} \partial_{t^{\prime}}^{m} K\left(t, t^{\prime}\right)
\end{aligned}
$$


Having defined $W\left[k_{n}\right]$ we turn to the issue of defining space-time fields. Let us go back to (4.2.59) for the moment. This defines (apart from some minor differences) the loop variable for a single string field. In this case $k(s)$ is not a function of $t$. A Taylor expansion in powers of $s$ yields all the vertex operators of the open string, but now at one point $z$. We consider thus the following definition:

$$
\begin{aligned}
\Phi(X(z+s)) & =\int\left[d k_{n}\right] e^{i k_{0} \cdot X(z)+\int d s k(s) \partial_{z} X(z+s)} \Phi(k(s)) \\
& =\int\left[d k_{n}\right] e^{i\left[k_{0} \cdot X(z)+k_{1} \partial_{z} X(z)+k_{2} \partial^{2} X(z)+\ldots+k_{n} \frac{\partial_{z}^{n} X(z)}{(n-1) !}+\ldots\right]} \Phi\left(k_{n}\right) \\
& =\int\left[d k_{n}\right] e^{i k_{0} \cdot X(z)}\left\{1+i k_{1} \partial_{z} X(z)+i k_{2} \partial^{2} X(z)-\right. \\
& \left.\frac{k_{1}^{\mu} k_{1}^{\nu}}{2} \partial_{z} X^{\mu}(z) \partial_{z} X^{\nu}(z)+\ldots\right\} \Phi\left(k_{n}\right)
\end{aligned}
$$

In the above expression $k_{0}$ is the usual momentum of the field. We have all the vertex operators corresponding to the various modes of the string. This $k_{1}^{\mu}$ is the analog of the polarization for $A^{\mu}\left(k_{0}\right)$. We can define

$$
\begin{aligned}
\left\langle k_{1}^{\mu}\right\rangle & =A^{\mu}\left(k_{0}\right) \\
\left\langle k_{2}^{\nu}\right\rangle & =S_{2}^{\mu}\left(k_{0}\right) \\
\left\langle k_{1}^{\mu} k_{1}^{\nu}\right\rangle & =S_{11}^{\mu \nu}\left(k_{0}\right)
\end{aligned}
$$

where $\langle\ldots\rangle=\int d k_{1} d k_{2} . . d k_{n} \ldots . . \Phi\left(k_{n}\right)$. (Note that $k_{0}$ is not integrated over.)

$k_{0}$ is just a number in this definition and one can insert as many powers of $k_{0}$ as one likes on both sides. Thus $\Phi(k(s))$ defines a map from the generalized momenta $k(s)$, in the loop variable, to space-time fields.

What is the relation between the string field $\Phi$ defined here and the wave-functional $\Psi$ defined in earlier sections? For the non-interacting case there is no difference. Thus when $t$ (and therefore $z(t))$ is fixed $\Psi\left(k_{n}\right)=$ $\Phi\left(k_{n}\right)$. However in the interacting case they are different because $\Phi$ always represents one string, whereas $\Psi$ stands for an arbitrary number of strings. An example will best illustrate this. Consider the field $A^{\mu}(X)$. An open string background represents an arbitrary number of fields. In the sigmamodel we insert the following boundary term into the functional integral:

$$
e^{i \int_{\partial \Gamma} d t A_{\mu}(X(t)) \partial_{t} X^{\mu}(t)}=1+i \int_{\partial \Gamma} d t A_{\mu}(X(t)) \partial_{t} X^{\mu}(t)
$$




$$
+\frac{1}{2 !}\left(i \int_{\partial \Gamma} d t A_{\mu}(X(t)) \partial_{t} X^{\mu}(t)\right)^{2}+\ldots
$$

$A^{\mu}$ is one mode of $\Phi$. On the RHS therefore we have an arbitrary number of fields, and therefore an arbitrary number of $\Phi$ 's. On the other hand this entire expression was written in Section 4 in terms of one wave-functional $\Psi$ in (4.3.66). $\Psi\left[k_{n}(t)\right]$ is a functional of $k_{n}(t)$. Thus by this device of making all the $k_{n}$ 's a function of $t$, we can go from the single string Hilbert space to the multi string Hilbert space. An explicit example of this was given in eqn (4.3.66).

Thus in (4.4.84) we have to replace $k_{n}$ by $k_{n}(t)$ [28, 27]. The loop variable becomes a "band" variable. Two $k_{n}$ 's with different values of $t$ thus represent two different strings - an interaction, whereas two $k_{n}$ 's with the same value of $t$ represent a higher excitation of the same string. This gives us

$$
\begin{aligned}
\left\langle k_{1}^{\mu}(t)\right\rangle & =A^{\mu}\left(k_{0}(t)\right) \\
\left\langle k_{2}^{\nu}(t)\right\rangle & =S_{2}^{\mu}\left(k_{0}(t)\right) \\
\left\langle k_{1}^{\mu}\left(t_{1}\right) k_{1}^{\nu}\left(t_{2}\right)\right\rangle & =S_{11}^{\mu \nu}\left(k_{0}\left(t_{1}\right)\right) \delta\left(t_{1}-t_{2}\right)+A^{\mu}\left(k_{0}\left(t_{1}\right)\right) A^{\nu}\left(k_{0}\left(t_{2}\right)\right)
\end{aligned}
$$

We also have to see the effect of $k_{0}(t) . k_{0}(t)$ represents the momentum of the string labelled by $t$.

Thus

$$
\left\langle k_{0}^{\nu}\left(t_{1}\right) k_{1}^{\mu}\left(t_{2}\right)\right\rangle=\delta\left(t_{1}-t_{2}\right) k_{0}^{\nu}\left(t_{1}\right) A^{\mu}\left(k_{0}\left(t_{1}\right)\right)
$$

and similarly

$$
\begin{gathered}
\left\langle k_{0}^{\rho}\left(t_{1}\right) k_{1}^{\mu}\left(t_{2}\right) k_{1}^{\nu}\left(t_{3}\right)\right\rangle=\delta\left(t_{1}-t_{2}\right) k_{0}^{\rho}\left(t_{2}\right) A^{\mu}\left(k_{0}\left(t_{1}\right)\right) A^{\nu}\left(k_{0}\left(t_{3}\right)\right) \\
+\delta\left(t_{1}-t_{3}\right) k_{0}^{\rho}\left(t_{3}\right) A^{\mu}\left(k_{0}\left(t_{1}\right)\right) A^{\nu}\left(k_{0}\left(t_{3}\right)\right)+\delta\left(t_{1}-t_{2}\right) \delta\left(t_{2}-t_{3}\right) k_{0}^{\rho}\left(t_{2}\right) S_{11}^{\mu \nu}\left(k_{0}\left(t_{2}\right)\right)
\end{gathered}
$$

Eqn (4.4.86) along with similar equations involving all the other $k_{n}$ 's, define the wave functional $\Psi$. For perturbative calculations we do not need an explicit expression for $\Psi$. We can thus calculate $\frac{d}{d \ln a}\left\langle O_{i}\right\rangle$ as a power series in the marginal fields in order to get the equations of motion. This method works for the tachyon and massless vector.

Note that for the tachyon $O_{i}=e^{i k . X}$, but due to momentum conservation,

$$
\left\langle e^{i k \cdot X}\right\rangle=0
$$

when $k \neq 0$. Thus the calculation starts at second order on bringing down one factor of $\int d z \int d k \phi(k) e^{i k \cdot X(Z)}$. Except for an overall integration 
over $z$ this is identical to the proper-time equation described in Section 3. The same is true for the (transverse) vector. However for massive modes this does not work due to issues of gauge invariance. One needs a more elaborate loop variable to get gauge invariant results. This is the subject of the next section.

\section{Gauge Invariance}

\section{$5.1 \quad$ Free Theory}

In order to obtain gauge invariant equations one needs a local version of the RG equations where $a$ is not constant on the world sheet. The following example illustrates this. One way to obtain the equations is to require that the cutoff dependence of operators vanish. Thus instead of evaluating $\left\langle O_{i}\right\rangle$, we just evaluate the cutoff dependence of $O_{i}$. If one doesn't impose momentum conservation on the VEV these two calculations are in fact the same. Thus for the tachyon, $\frac{1}{a} e^{i k . X}=e^{\left(\frac{k^{2}}{2 \pi}-1\right) l n a}: e^{i k . X}:$. Here $: . .:$ denotes normal ordering. Setting $\frac{d}{d \ln a}=0$ gives the equation of motion of the tachyon. Let us attempt to do this for the vector.

$$
A^{\mu}(k) \partial_{z} X^{\mu} e^{i k . X}=-i k . A(k) \frac{\partial_{z} \sigma}{2 \pi}: e^{i k . X}: e^{\frac{k^{2}}{2 \pi} \sigma}+A^{\mu}(k): \partial_{z} X^{\mu} e^{i k . X}: e^{\frac{k^{2}}{2 \pi} \sigma}
$$

where we have used $\sigma$ in place of $\ln a$ and also $\left\langle\partial_{z} X(z) X(z)\right\rangle=\frac{\partial_{z} \sigma}{2 \pi} . \sigma$ can be thought of as the Liouville mode which is a local measure of the scale. We can say that $a \rightarrow a e^{\sigma}$. Now we vary w.r.t $\sigma$, and after integrating by parts, set $\sigma=0$ to find,

$$
\left(-k \cdot A(k) k^{\mu}+k^{2} A^{\mu}(k)\right): \partial_{z} X^{\mu} e^{i k \cdot X}:
$$

This is Maxwell's equation. Had we not kept the $\partial_{z} \sigma$ piece we would have obtained only one of the terms and the result would not have been gauge-invariant. This illustrates the importance of local scale invariance.

We can attempt to do the same thing for the massive modes of the form $S_{2} \partial_{z}^{2} X e^{i k . X}$. In that case one can expect terms of the form $k \cdot S(k) \partial_{z}^{2} \sigma$ : $e^{i k . X}:$. On varying w.r.t $\sigma$, when we integrate by parts and differente $e^{i k . X}$ twice, we get terms of the form $k . S(k) k^{\mu} k^{\nu}: \partial_{z} X^{\mu} \partial_{z} X^{\nu} e^{i k . X}:$. This term is clearly unacceptable in an equation of motion for a free field because of the presence of three factors of $k$. The problem gets worse at higher mass levels. Thus we have to modify our loop variable [26]. 
The basic idea introduced in [26] is to introduce additional variables $x_{n}, n>0$ that, very roughly, have the property that $\frac{\partial}{\partial x_{n}} \approx \partial_{z}^{n}$. This means that when we integrate by parts over $x_{n}$ (not $z$ ) and the derivative acts on $e^{i k . X}$ we never bring down more than one power of $k$.

Another problem with the loop variable is that it depends on the parametrization of $s$. It is not diffeomorphism invariant. This can be rectified by introducing an einbein along the loop. The modes of this einbein also provide the extra variables $x_{n}$. Thus both problems are solved.

We thus consider the following loop variable:

$$
e^{i \int_{c} \alpha(s) k(s) \partial_{z} X(z+s) d s+i k_{0} X(z)}
$$

$\alpha(s)$ is an einbein. Let us assume the following Laurent expansion:

$$
\alpha(s)=1+\frac{\alpha_{1}}{s}+\frac{\alpha_{2}}{s^{2}}+\frac{\alpha_{3}}{s^{3}}+\ldots
$$

Let us define

$$
\begin{aligned}
Y & =X+\alpha_{1} \partial_{z} X+\alpha_{2} \partial_{z}^{2} X+\alpha_{3} \frac{\partial_{z}^{3} X}{2}+\ldots+\frac{\alpha_{n} \partial_{z}^{n} X}{(n-1) !}+\ldots \\
& =X+\sum_{n>0} \alpha_{n} \tilde{Y}_{n} \\
Y_{1} & =\partial_{z} X+\alpha_{1} \partial_{z}^{2} X+\alpha_{2} \frac{\partial_{z}^{3} X}{2}+\ldots+\frac{\alpha_{n-1} \partial_{z}^{n} X}{(n-1) !}+\ldots \\
\ldots & \cdots \\
Y_{m} & =\frac{\partial_{z}^{m} X}{(m-1) !}+\sum_{n>m} \frac{\alpha_{n-m} \partial_{z}^{n} X}{(n-1) !}
\end{aligned}
$$

If we define $\alpha_{0}=1$ then the $>$ signs in the summations above can be replaced by $\geq$.

Using these equations one can write

$$
e^{i \int_{c} \alpha(s) k(s) \partial_{z} X(z+s) d s+i k_{0} X(z)}=e^{i \sum_{n} k_{n} Y_{n}(z)}
$$

It is understood that $Y_{0}=Y$.

Let us now introduce $x_{n}$ by the following:

$$
\alpha(s)=\sum_{n \geq 0} \alpha_{n} s^{-n}=e^{\sum_{m \geq 0} s^{-m} x_{m}}
$$


Thus

$$
\begin{aligned}
\alpha_{1} & =x_{1} \\
\alpha_{2} & =\frac{x_{1}^{2}}{2}+x_{2} \\
\alpha_{3} & =\frac{x_{1}^{3}}{3 !}+x_{1} x_{2}+x_{3}
\end{aligned}
$$

They satisfy the property,

$$
\frac{\partial \alpha_{n}}{\partial x_{m}}=\alpha_{n-m}, \quad n \geq m
$$

Using this we see that

$$
Y_{n}=\frac{\partial Y}{\partial x_{n}}
$$

Now we can do the same operation of imposing $\frac{\delta}{\delta \sigma}=0$. Except we will define $\Sigma=\langle Y(z) Y(z)\rangle$. This is equal to the previous $\sigma$ in coordinates where $\alpha(s)=1$. Thus we have for the coincident two point functions:

$$
\begin{aligned}
\langle Y Y\rangle & =\Sigma \\
\left\langle Y_{n} Y\right\rangle & =\frac{1}{2} \frac{\partial \Sigma}{\partial x_{n}} \\
\left\langle Y_{n} Y_{m}\right\rangle & =\frac{1}{2}\left(\frac{\partial^{2} \Sigma}{\partial x_{n} \partial x_{m}}-\frac{\partial \Sigma}{\partial x_{n+m}}\right)
\end{aligned}
$$

Using this the normal ordering gives:

$$
\begin{aligned}
e^{i \int_{c} \alpha(s) k(s) \partial_{z} X(z+s) d s+i k_{0} X(z)=} & e^{i \sum_{n} k_{n} Y_{n}(z)} \\
= & \exp \left\{k_{0}^{2} \Sigma+\sum_{n>0} k_{n} \cdot k_{0} \frac{\partial \Sigma}{\partial x_{n}}+\right. \\
& \left.\sum_{n, m>0} k_{n} \cdot k_{m} \frac{1}{2}\left(\frac{\partial^{2} \Sigma}{\partial x_{n} \partial x_{m}}-\frac{\partial \Sigma}{\partial x_{n+m}}\right)\right\} \\
& : e^{i \sum_{n} k_{n} Y_{n}(z)}:
\end{aligned}
$$

We can now operate with $\frac{\delta}{\delta \Sigma}$ and set $\Sigma=0$. We will only give one sample variation here:

$$
\frac{\delta}{\delta \Sigma}\left[k_{n} . k_{m} \frac{1}{2}\left(\frac{\partial^{2} \Sigma}{\partial x_{n} \partial x_{m}}-\frac{\partial \Sigma}{\partial x_{n+m}}\right)\right]: e^{i k_{0} . Y}:=:\left(\frac{1}{2} i k_{0}^{\mu} i k_{0}^{\nu} Y_{n}^{\mu} Y_{m}^{\nu}+i k_{0}^{\mu} Y_{n+m}^{\mu}\right) e^{i k_{0} \cdot Y}:
$$


One can thus collect all the coefficients of a particular vertex operator, say : $Y_{n}^{\mu} e^{i k_{0} . Y}$ :, and this gives the free equation of motion. Note that they never contain more than two space-time derivatives. This solves the first problem.

The second problem is that of gauge invariance. We have assumed that $\alpha(s)$ is being integrated over, which is why we are allowed to integrate by parts. This means that

$$
k(s) \rightarrow \lambda(s) k(s)
$$

is equivalent to $\alpha(s) \rightarrow \lambda(s) \alpha(s)$, which is clearly just a change of an integration variable. Assuming the measure is invariant this does nothing to the integral. The measure $\mathcal{D} \alpha(s)$ has been chosen to be $\prod_{n} d x_{n}$. If we set $\lambda(s)=e^{\sum_{m} y_{m} s^{-m}}$, then the gauge transformation (5.1.101) is just a translation, $x_{n} \rightarrow x_{n}+y_{n}$ which leaves the measure invariant. Thus we conclude that (5.1.101) gives the gauge transformation.

If we expand $\lambda(s)$ in inverse powers of $s$

$$
\lambda(s)=\sum_{n} \lambda_{n} s^{-n}
$$

Then we can write (5.1.101) as

$$
k_{n} \rightarrow \sum_{m=0}^{n} \lambda_{m} k_{n-m}
$$

We set $\lambda_{0}=1$.

In order to interpret these equations in terms of space-time fields we use (4.4.84). They have to be extended to include $\lambda$. Thus we assume that the string wave-functional is also a functional of $\lambda(s)$. Thus we set

$$
\begin{aligned}
\left\langle\lambda_{1}\right\rangle & =\Lambda_{1}\left(k_{0}\right) \\
\left\langle\lambda_{1} k_{1}^{\mu}\right\rangle & =\Lambda_{11}^{\mu}\left(k_{0}\right) \\
\left\langle\lambda_{2}\right\rangle & =\Lambda_{2}\left(k_{0}\right)
\end{aligned}
$$

The gauge transformations (5.1.102) thus become on mapping to space time fields by evaluating $\langle.$.$\rangle :$

$$
\begin{aligned}
A^{\mu}\left(k_{0}\right) & \rightarrow A^{\mu}(k o)+k_{0}^{\mu} \Lambda_{1}\left(k_{0}\right) \\
S_{2}^{\mu}\left(k_{0}\right) & \rightarrow S_{2}^{\mu}(k o)+k_{0}^{\mu} \Lambda_{2}\left(k_{0}\right)+\Lambda_{11}^{\mu} \\
S_{11}^{\mu \nu} & \rightarrow S_{11}^{\mu \nu}+k_{0}^{(\mu} \Lambda_{11}^{\nu)}
\end{aligned}
$$


These are more or less the canonical gauge transfomations for a massive spin two field. [3 Now it is known that the gauge transformation parameters of higher spin fields obey a certain tracelessness condition [34, 35]. We will see this below also.

When one actually performs the gauge transformations we find the following mechanism for gauge invariance. It changes the normal ordered loop variable by a total derivative in $x_{n}$ which doesn't affect the equation of motion. More precisely the gauge variation of the loop variable is a term of the form $\frac{d}{d x_{n}}[A(\Sigma) B]$, where $B$ doesn't depend on $\Sigma$. The coefficient of $\delta \Sigma$ is obtained as

$$
\begin{gathered}
\int \delta\left(\frac{d}{d x_{n}}[A(\Sigma) B]\right)=\int\left(\frac{d}{d x_{n}}\left(\frac{\delta A}{\delta \Sigma} \delta \Sigma\right) B+\frac{\delta A}{\delta \Sigma} \delta \Sigma \frac{d B}{d x_{n}}\right) \\
=\int\left[-\frac{\delta A}{\delta \Sigma} \frac{d B}{d x_{n}}+\frac{\delta A}{\delta \Sigma} \frac{d B}{d x_{n}}\right] \delta \Sigma=0
\end{gathered}
$$

Here we have used an integration by parts.

Actually one finds on explicit calculation that the variation is not a total derivative. This is because in deriving (5.1.99) some identities have been used. Thus only if we use those identities in the variation will we be able to write the variation as a total derivative. However we do not want to use them because we would like to leave $\Sigma$ unconstrained when we vary. Thus constraints have to be imposed elsewhere. It can easily be checked that the terms that have to be put to zero are all of the form

$$
\lambda_{n} k_{m} \cdot k_{p} \cdots
$$

where $\ldots$ refers to any other factors of $k_{m}$ [26, 33]. Thus all traces of gauge parameters have to be set to zero. This thus explains the tracelessness mentioned earlier.

In [26, 33] some examples, namely spin-2 and spin-3 are explicitly worked out.

The form of the gauge transformation (5.1.101) is very suggestive: It is a scale transformation in space-time. It is local along the loop. As explained in 26] getting a gauge transformation of this type was one of the original motivations for this formalism.

\footnotetext{
${ }^{3}$ They become identical after we perform a dimensional reduction. This will be described later.
} 


\subsection{Dimensional Reduction}

In the gauge invariant formalism we have not made contact with string theory because we have not reproduced the mass spectrum. In earlier sections we were dealing with the physical states, and the mass, being the dimension of the operator, came out right because there are always appropriate powers of the cutoff $a$ multiplying the operator to give it the right dimension. In the gauge invariant formalism, we are using the new $\Sigma$ field, which is a complicated combination of the old $\sigma$ field and its derivatives. Thus we cannot just introduce it by replacing $a$ by $a e^{\sigma}$, as one would normally do when introducing the Liouville mode. We will introduce it by hand as a variant of the Kaluza-Klein mechanism. Thus we will let the momentum $k_{0}^{\mu}$ be a 27-dimensional vector rather than a 26-dimensional one. We will let $k_{0}^{26}$ stand for the mass as in Kaluza-Klein theories but instead of letting $k_{0}$ be multiples of $\frac{1}{R}$ we will assume that $k_{0}^{2}$ is a multiple of $\frac{1}{R^{2}}$. Furthermore gauge invariance will force us to make all the $k_{n}$ 's 27-dimensional. This means many new modes are being introduced into the theory. This is just as well - we know that the gauge invariant formalism requires an additional bosonic coordinate worth of modes [36].

We denote $k^{26}$ by $q$. We set $q_{0}$ to $\sqrt{(P-1)}$, where $P$ is the engineering dimension of the vertex operator. Thus for the tachyon $P=0$, for the vector $P=1$ etc. Finally there is one subtlety. The first oscilator of the extra bosonic coordinate was set to zero in [36. In our case the first mode $q_{1}$ will not be set to zero identically because that would violate gauge invariance. We will impose relations of the form:

$$
\begin{gathered}
\left\langle q_{1}\right\rangle=0 . \\
\left\langle q_{1} q_{1}\right\rangle=\left\langle q_{2} q_{0}\right\rangle=S_{2} ;\left\langle\lambda_{1} q_{1}\right\rangle=\left\langle\lambda_{2} q_{0}\right\rangle=\Lambda_{2} . \\
\left\langle q_{1} k_{1}^{\mu}\right\rangle=\left\langle k_{2}^{\nu} q_{0}\right\rangle=S_{2}^{\mu} .
\end{gathered}
$$

Note that $q_{0}$ is 1 for the spin- 2 field. There will relations of this type that will enable us to get rid of $q_{1}$ completely. The form of the relations is such as to maintain gauge invariance. They can be built up recursively.

We merely summarize the results for the massive spin-2 field:

$$
\begin{gathered}
\delta S_{11}^{\mu \nu}=k_{0}^{(\mu} \Lambda^{\nu)} \\
\delta S_{2}^{\mu}=\Lambda_{11}^{\mu}+k_{0}^{\mu} \Lambda_{2} \\
\delta S_{2}=2 \Lambda_{2}
\end{gathered}
$$


These are in the "standard" form, where the extra auxiliary fields $S_{2}$ and $S_{2}^{\mu}$ can be set to zero to recover the Pauli-Fierz equations for massive spin-2 fields. Further details can be found in [26] and references therein.

\section{$5.3 \quad$ Interactions}

We now turn to the all important issue of introducing interactions. The prescription for interactions that was first proposed in [28], worked out in [29, 30], and modified into a very simple form in [32] is to introduce an extra parameter $t$ in all the variables. Thus $k(s) \rightarrow k(s, t)$ and $X(z+s) \rightarrow X(z(t)+$ $s) . t$ is supposed to label the different strings. This was an ad-hoc procedure then. But in view of the calculations described in Section 4 where we defined the partition function in terms of a generating functional $W[k(s, t)]$ (see eqn (4.4.80), in retrospect the meaning of introducing $t$ is very clear. As the discussion following (5.3.114) makes clear we end up calculating $W[k(s, t)]$. Thus it is clear that this procedure gives interactions. What we have to ensure is that gauge transformations can still be consistently defined.

Let us follow the prescription of [28, 29, 30]. First of all we need a generalization of $\Sigma$ for the interacting case. This can be done by considering an alternative definition of $\Sigma$ [39, 29].

Perform a general conformal transformation, with group element $e^{\sum_{n} \lambda_{-n} L_{+n}}$, on the loop variable:

$$
e^{i \sum_{n} k_{n} Y_{n}(z)} .
$$

The resulting $\lambda$ dependence (which is also an expression of the $\sigma$ (Liouville mode) dependence), can be rewritten in the form given in (5.1.100).

We use the following result [40]:

$$
e^{\sum_{n} \lambda_{-n} L_{+n}} e^{i K_{m} \tilde{Y}_{m}}=e^{K_{n} \cdot K_{m} \lambda_{-n-m}+\tilde{Y_{n}} \tilde{Y_{m}} \lambda_{+n+m}+i m K_{n} \tilde{Y_{m} \lambda_{-n+m}}} e^{i K_{m} \tilde{Y_{m}}}
$$

The anomalous term is $K_{n} \cdot K_{m} \lambda_{-n-m}$ and the classical term is $m K_{n} \tilde{Y}_{m} \lambda_{-n+m}$. We will ignore the classical piece: this can be rewritten as a $(\text { mass })^{2}$ term, which will be reproduced by performing a dimensional reduction, and other pieces involving derivatives of $\Sigma$ (defined below), which correspond to field redefinitions [26]. We can apply (5.3.109) to the loop variable (5.3.108) by setting

$$
K_{m}=\sum_{n} k_{m-n} \alpha_{n}
$$


Defining

$$
\Sigma=\sum_{p, q} \alpha_{p} \alpha_{q} \lambda_{-p-q}
$$

we recover (5.1.100). This definition of $\Sigma$ generalizes in a straightforward way to the interacting case.

The $\left.\Sigma\left(z_{1}, z_{2}\right)\right)$ will be defined in terms of $\rho\left(z_{1}, z_{2}\right)$, which is defined as follows. First

$$
e^{: \frac{1}{2} \int d u \lambda(u)\left[\partial_{u} X(u)\right]^{2}:} e^{i k_{n} \frac{\partial_{z_{1}}^{n}}{(n-1) !} X\left(z_{1}\right)} e^{i p_{m} \frac{\partial_{z_{2}}^{m}}{(m-1) !} X\left(z_{2}\right)}
$$

defines the action of the Virasoro generators on the two sets of vertex operators.

$$
\begin{gathered}
=e^{k_{n} \cdot p_{m}} \frac{\partial_{z_{1}}^{n}}{(n-1) !} \frac{\partial_{z_{2}}^{m}}{(m-1) !} \oint d u \frac{\lambda(u)}{z_{1}-z_{2}}\left[\frac{1}{z_{1}-u}-\frac{1}{z_{2}-u}\right] \\
=e^{k_{n} \cdot p_{m} \frac{\partial_{z_{1}}^{n}}{(n-1) !} \frac{\partial_{z_{2}}^{m}}{(m-1) !} \rho\left(t, t^{\prime}\right)} \\
=e^{k_{n} \cdot p_{m} \rho_{n, m}\left(z_{1}, z_{2}\right)}
\end{gathered}
$$

where $\rho\left(z_{1}, z_{2}\right)=\frac{\lambda\left(z_{1}\right)-\lambda\left(z_{2}\right)}{z_{1}-z_{2}} . \quad \rho$ is a generalization of the usual Liouville mode $\sigma=\frac{d \lambda}{d z}$, for the case where the vertex operators are not located at the same point. It has to be further modified to make it gauge covariant - this will give us $\Sigma$. We will do this later.

In the interacting case when we consider the normal ordering of the products of vertex operators, we not only have in the exponent, $\rho$ and its derivatives, but also $K$ (Green's function) and its derivatives. As a simple illustration consider the conventional normal ordering of the product $e^{i k . X(z)+i p . X(w)}$,

$$
\begin{aligned}
e^{i k \cdot X(z)+i p \cdot X(w)} & =e^{\frac{1}{2}\langle(i k \cdot X(z)+i p \cdot X(w))(i k \cdot X(z)+i p \cdot X(w))\rangle}: e^{i k \cdot X(z)+i p \cdot X(w)}: \\
& =e^{\left\{\frac{1}{2 \pi}\left[\left(k^{2}+p^{2}\right) \ln \left(a e^{\sigma}\right)+2 k \cdot p \ln (z-w)\right]\right.} \\
& =e^{\left\{\frac{1}{2 \pi}\left[\left(k^{2}+p^{2}\right) \ln (a)+2 k \cdot p \ln (z-w)+\left(k^{2}+p^{2}\right) \sigma\right]\right.}
\end{aligned}
$$

In the exponent $\sigma$ is what is generalized to $\rho$. But we also have $K(z, w)=$ $\ln (z-w)$. In $a$ is just $K(z, z)$ regularized. A convenient form for $K$ that can be used is $K(z, w)=\frac{1}{2 \pi} \ln \left(a^{2}+(z-w)^{2}\right)$.

Notice also that if we denote by $V(k, z)$ the vertex operator at $z$ with momentum $k$, the above equation (5.3.114) can be written as

$$
V(k, z) V(p, w)=\langle V(k, z) V(p, w)\rangle: V(k, z) V(p, w):
$$


The only subtle point is that there is effectively a momentum conserving delta-function in (5.3.115), whereas it is not there in (5.3.114).

Thus if our loop variables are of the form

$$
e^{i \int d t \sum_{n \geq 0} K_{n}(t) \tilde{Y}_{n}(t)}
$$

we get for the normal ordered expression, the following $\rho, K$-dependence:

$$
\begin{gathered}
\exp \left\{\int d t \int d t ^ { \prime } \left[K_{0}(t) \cdot K_{0}\left(t^{\prime}\right)\left[K\left(t, t^{\prime}\right)+\rho\left(t, t^{\prime}\right)\right]+\right.\right. \\
\left.\left.\sum_{n>0} 2 K_{n}(t) \cdot K_{0}\left(t^{\prime}\right)\left[K_{n, 0}\left(t, t^{\prime}\right)+\rho_{n, 0}\left(t, t^{\prime}\right)\right]+\sum_{n, m>0} K_{n}(t) \cdot K_{m}\left(t^{\prime}\right)\left[K_{n, m}\left(t, t^{\prime}\right)+\rho_{n, m}\left(t, t^{\prime}\right)\right]\right]\right\} \\
: \exp \left(i \int d t K_{n}(t) \tilde{Y}_{n}(t)\right):
\end{gathered}
$$

As in (5.3.115), the coefficient of the normal ordered operator is

$$
\left\langle e^{i \int d t \sum_{n \geq 0} K_{n}(t) \tilde{Y}_{n}(t)}\right\rangle
$$

This is of course nothing but the generating functional $W\left[K_{n}(t)\right]$ ! Thus our prescription of introducing $t$-dependence, makes contact with our earlier discussions on interacting string theory in Section 4.

Now one has to introduce the $\alpha_{n}$ 's to make the formalism gauge invariant. At this point the method adopted in [28, 29, 30] is to introduce $\alpha_{n}(t)$ and define a generalized Green's function, $G\left(t, t^{\prime}\right)$, and generalized Liouville mode $\Sigma\left(t, t^{\prime}\right)$ by taking combinations of the form $\sum_{n, m} \alpha(t)_{p-n} \alpha\left(t^{\prime}\right)_{r-m}[K+$ $\rho]_{n, m}\left(t, t^{\prime}\right)=[G+\Sigma]_{r, p}\left(t, t^{\prime}\right)$. The technical complication involved in this is that $\Sigma\left(t, t^{\prime}\right)$ is not a local field but a bi-local field. A Taylor expansion was then performed. There is also some ambiguity regarding the $t$-dependence of $\alpha$. In [29, 30] the $t$-dependence was retained in the intermediate stages of the calculation but in the end the $\alpha$ 's were set to be $t$-independent.

A simpler alternative was followed in [32] which we will use here. All ambiguities are avoided by first Taylor expanding all the $X(z)$ 's about $X(0)$ in powers of $z$. Thus all calculations involve the vertex operators located at a single point $z=0$. A This is well defined provided we keep a finite cutoff in all intermediate stages of the calculation so that coincident two-point functions are finite. Thus we could use for instance $K\left(t, t^{\prime}\right)=\frac{1}{2 \pi} \ln \left(a^{2}+\left(t-t^{\prime}\right)^{2}\right)$. Since the equations involve off-shell vertex operators, in any case we do need a finite cutoff.

\footnotetext{
${ }^{4}$ The choice $z=0$ is not important - it could have been about any point $z=z_{0}$ as well.
} 
Thus we first write

$$
\sum_{n \geq 0} k_{n}(t) \tilde{Y}_{n}(z(t))=\sum_{n \geq 0} \bar{k}_{n}(t,-z(t)) \tilde{Y}_{n}(0)
$$

The argument $t$ is superfluous and one can write $\bar{k}_{n}(t,-z(t))=\bar{k}_{n}(-z(t))$. If we let $z(t)=t$ we can further simplify notation and write just $\bar{k}_{n}(-t)$.

This defines $\bar{k}_{n}(-z(t))$ to be

$$
\bar{k}_{q}(-z(t))=\sum_{n=0}^{n=q} k_{n}(t) D_{n}^{q}(z(t))^{q-n}
$$

where

$$
\begin{aligned}
D_{n}^{q} & ={ }^{q-1} C_{n-1}, \quad n, q \geq 1 \\
& =\frac{1}{q}, \quad n=0 \\
& =1, \quad n=q=0
\end{aligned}
$$

Note that $\bar{k}_{0}(-z(t))=k_{0}(t)$.

Now we can write the gauge invariant loop variable as

$$
e^{\int d t \sum_{n \geq 0} i \bar{k}_{n}(-z(t)) Y_{n}(0)}
$$

One can also rewrite this as a loop variable analogous to (5.1.89). Define first, $k(s-z)=\sum_{n \geq 0} k_{n}(-z) s^{-n}$. Consider

$$
\sum_{n>0} k_{n}(-z) \tilde{Y}_{n}(0)+k_{0} X(z)=\sum_{n>0}\left(k_{n}(-z)+k_{0} \frac{z^{n}}{n}\right) \tilde{Y}_{n}(0)+k_{0} \tilde{Y}(0)
$$

$(\tilde{Y} \equiv X$.$) (The variable in brackets is in fact \bar{k}_{n}(-z)$ defined earlier in (5.3.119.)

$$
\begin{aligned}
& =\sum_{n>0} \bar{k}_{n}(-z) \tilde{Y}_{n}(0)+k_{0}(z) X(0) \\
& =\int d s \sum_{n>0} \bar{k}_{n}(-z) s^{-n} \partial X(s)+k_{0}(z) X(0) \\
& =\int d s \bar{k}(s,-z) \partial X(s)+k_{0}(z) X(0)
\end{aligned}
$$


This equation defines $\bar{k}(s,-z)$. We can now introduce an einbein $\alpha(s)$ to get

$$
\int d s \bar{k}(s,-z) \alpha(s) \partial X(s)+k_{0}(z) X(0)
$$

Thus (5.3.121) (or 5.3.123)) is the loop variable we work with. The interesting thing is that all the $z$-dependence is in $k$ rather than in $X$. Thus we have to work with $Y_{n}(0)$. There is no ambiguity about the $t$-dependence of $\alpha_{n}$ since they are all at one point. Thus (5.3.121) looks a lot like (5.1.100) except that the coefficients $k_{n}$ are replaced by $\int d t \bar{k}_{n}(-t)$, and $\Sigma$ replaced by $\Sigma(0,0)+G(0,0)$. This also makes the calculations very similar to the free case. Only after calculating $W\left[k_{n}(t)\right]$ when we integrate over $\Psi\left[k_{n}(t)\right]$ the effects of this replacement will show up through all the $t$-dependences of the correlation functions and subsequent integrations over $t$ [32]. This will be shown below.

Thus the normal ordering gives exactly the same result as (5.1.100) with appropriate replacements:

$$
\begin{aligned}
e^{\int d t \sum_{n \geq 0} i \bar{k}_{n}(-t) Y_{n}(0)=} & \exp \left\{\int d t \int d t^{\prime} \bar{k}_{0}(-t) \bar{k}_{0}\left(-t^{\prime}\right)(\Sigma(0,0)+G(0,0))+\right. \\
& \sum_{n>0} \bar{k}_{n}(-t) \cdot \bar{k}_{0}\left(-t^{\prime}\right) \frac{\partial(\Sigma(0,0)+G(0,0))}{\partial x_{n}}+ \\
& \sum_{n, m>0} \bar{k}_{n}(-t) \cdot \bar{k}_{m}\left(-t^{\prime}\right) \frac{1}{2}\left(\frac{\partial^{2}(\Sigma(0,0)+G(0,0))}{\partial x_{n} \partial x_{m}}-\right. \\
& \left.\left.\frac{\partial(\Sigma(0,0)+G(0,0))}{\partial x_{n+m}}\right)\right\} \\
& : e^{\int d t \sum_{n \geq 0} i \bar{k}_{n}(-t) Y_{n}(0)}: \\
= & W\left[k_{n}(t)\right]: e^{\int d t \sum_{n \geq 0} i \bar{k}_{n}(-t) Y_{n}(0)}:
\end{aligned}
$$

We have already specialized to $z(t)=t$ in the above equation.

The equations of motion can be obtained as in the free case by operating with $\frac{\delta}{\delta \Sigma(0,0)}$ and then setting $\Sigma$ to zero. One can also set $\Sigma=0$ right in the beginning and perform the operation $\frac{\delta}{\delta G(0,0)}$ treating $G$ formally as a field and we get the same answer. Either way we can see that this is a gauge covariantized version of $\frac{\delta}{\delta \sigma}$ (or $\frac{d}{d \ln a}$ ), which is the renormalization group operator. This also makes contact with the formalism of Section 2 and Section 3. 
We will now define gauge transformations:

$$
\bar{k}_{n}(-t) \rightarrow \bar{k}_{n}(-t)+\int d t^{\prime} \sum_{m \leq n} \lambda_{m}\left(t^{\prime}\right) \bar{k}_{n-m}(-t)
$$

This is the same as in the free case except for the replacent of $\lambda_{n}$ by $\int d t \lambda_{n}(t)$. Thus the gauge invariance of the equations follows in exactly the same way as in the free case. Note that we also have the interacting version of the constraints, which now have the form:

$$
\int d t \lambda_{p}(t) \bar{k}_{n}\left(t_{1},-z\left(t_{1}\right)\right) \cdot \bar{k}_{m}\left(t_{2},-z\left(t_{2}\right)\right) \ldots=0
$$

There will also be equations generalizing (4.4.86) to include $\lambda_{n}(t)$ (apply the definitions (5.1.103). We do not write them out explicitly.

\subsection{Defining Gauge Transformations of Space-Time Fields}

Let us say that $\mathcal{M}$ is a map from an expression $L$ involving products of $\bar{k}_{n}(-t)$ to an expression $S$ involving products of space-time fields.

Thus

$$
\mathcal{M}: L \longrightarrow S
$$

This is the operation $\langle\ldots\rangle=\int \mathcal{D} k_{n} \mathcal{D} \lambda_{n} \ldots \Psi\left[k_{n}(t), \lambda_{n}(t)\right]$.

Let $\mathcal{G}$ denote the gauge transformation (5.3.125). Thus

$$
\mathcal{G}: L \quad \longrightarrow \quad L^{g}
$$

Then $\mathcal{M}$ maps this to another set of space-time fields - $S^{g}$.

$$
\mathcal{M G}: L=S^{g}
$$

Let $G$ be the gauge transformation on $S$ induced by this. Thus

$$
G: S \longrightarrow \mathcal{M G} L=S^{g}
$$

If we define gauge transformations this way we are guaranteed that any expression $L$ that is invariant under $\mathcal{G}$, (i.e. $L_{g}=0$ ) maps to a gauge invariant $S$, (i.e. $S_{g}=0$ ). This would mean that all the loop variable equations that are by construction gauge invariant will lead to gauge invariant equations of motion. The question is whether there is a unique and well defined action of $G$ on individual fields such that (5.4.126) (which involves sums of products of space-time fields) is satisfied. 
We show that the answer is in the affirmative. Let us be more precise and specify the steps involved in the argument. We will fill in the details afterwards.

\section{Step 1}

Any equation of motion is a sum of terms of the type

$$
\begin{aligned}
\left\langle\bar{k}_{n_{1}}^{\mu_{1}}\left(-t_{1}\right) \bar{k}_{n_{2}}^{\mu_{2}}\left(-t_{2}\right) \bar{k}_{n_{3}}^{\mu_{3}}\left(-t_{3}\right) \ldots \bar{k}_{n_{r}}^{\mu_{r}}\left(-t_{r}\right)\right\rangle & =\left\langle L_{n_{1}, n_{2}, \ldots, n_{r}}\right\rangle \\
& =S_{n_{1}, n_{2}, \ldots, n_{r}}
\end{aligned}
$$

where $S_{n_{1}, n_{2}, \ldots, n_{r}}$ is a sum of products of space-time fields and also various powers of $t_{i}$. (All the $t_{i}$ are integrated at the end of the day.) Note that the set of numbers uniquely specify $L_{n_{1}, n_{2}, \ldots, n_{r}}$ and vice versa. f

\section{Step 2}

It is possible to define uniquely the action of $G$ on space-time fields so that the gauge transformation $G$ acting on this expression $S_{n_{1}, n_{2}, \ldots, n_{r}}$ is the expression $S_{g}$ obtained by the action of $\mathcal{M G}$ on $L_{n_{1}, n_{2}, \ldots, n_{r}}$ as explained above. That is

$$
G S_{n_{1}, n_{2}, \ldots, n_{r}}=S_{n_{1}, n_{2}, \ldots, n_{r}}^{g} \equiv\left\langle\mathcal{G} L_{n_{1}, n_{2}, \ldots, n_{r}}\right\rangle \equiv\left\langle L_{n_{1}, n_{2}, \ldots, n_{r}}^{g}\right\rangle
$$

\section{Step 3}

The loop variable equation of motion is of the form $L_{1}+L_{2}+\ldots$ by step 1 . If $\mathcal{G}\left(L_{1}+L_{2}+\ldots\right)=L_{1}^{g}+L_{2}^{g}+. .=0$ then $G\left(S_{1}+S_{2}+S_{3}+..\right)=S_{1}^{g}+S_{2}^{g}+. . .=0$. Thus the equations are gauge invariant in terms of space-time fields.

Let us fill in the details:

Step 1:

The expression $W\left[k_{n}(t)\right]: e^{\int d t \sum_{n \geq 0} i \bar{k}_{n}(-t) Y_{n}(0)}$ : eqn. (5.3.124), is clearly of the type $L_{n_{1}, n_{2}, \ldots, n_{r}}$ (and its Lorentz index contractions.) When we vary w.r.t. $\Sigma$ we integrate by parts on $x_{n}$. But $L_{n_{1}, n_{2}, \ldots, n_{r}}$ does not depend on $x_{n}$ so it is unaffected. Thus every equation is made up of terms $L_{n_{1}, n_{2}, \ldots, n_{r}}$. This ensures that the set of numbers uniquely specifies the combination that occurs in the equation of motion. Thus we get also uniquely $S_{n_{1}, n_{2}, \ldots, n_{r}}$.

Step 2:

This is the crucial step.

A given set of numbers $n_{1}, \ldots n_{r}$ uniquely defines the set of terms involved in $S_{n_{1}, n_{2}, \ldots, n_{r}}$. It involves one highest level field that we can call $S_{n_{1}, n_{2}, n_{3} . . n_{r}}^{\mu_{1} \mu_{2} \mu_{3} \ldots \mu_{r}}$ and products of lower level fields such as $S_{n_{1}, n_{2}}^{\mu_{1} \mu_{2}} S_{n_{3}, \ldots n_{r}}^{\mu_{3} \ldots \mu_{r}}$. If some of the

\footnotetext{
${ }^{5}$ There could be Lorentz-index contractions. This is trivially taken care of.
} 
$n_{i}$ are zero then we consider an expression $S^{\prime}$ without those $n_{i}$. Then $S$ is obtained from $S^{\prime}$ by taking space-time derivatives. This is because the operation $\int d t k_{0}(t)$ is just differentiation. So we assume that none of the $n_{i}$ are zero.

Given the expression $S_{n_{1}, n_{2}, \ldots, n_{r}}^{g}$, we can define recursively the gauge transform of the highest level field $S_{n_{1}, n_{2}, n_{3} . n_{r}}^{\mu_{1} \mu_{2} \mu_{3} \ldots \mu_{r}}$, as $S_{n_{1}, n_{2}, \ldots, n_{r}}^{g}$ minus the gauge transform of the rest of the expression which involves only lower level fields. This definition is unique (assuming the lower level fields have uniquely defined transformations). This procedure therefore allows us to recursively define gauge transforms of higher fields in terms of lower ones such that $S^{g}=G S$.

Step 3:

Follows from the definition of $S^{g}$.

What we really have is an algorithm for defining gauge transformations of fields in such a way that the equations of motion are gauge invariant.

An example illustrating this is given in [32]. We now turn to the issue of dimensional reduction in the interacting case.

\subsection{Dimensional Reduction in the Interacting Case}

In the free theory we set $q_{0}=\sqrt{P-1}$. In the interacting case we proceed as follows. Concentrate on the transverse physical state scattering where all the particles are on shell. We set $q_{0}\left(t_{i}\right)$ to the value that produces the correct scattering amplitude. Then we will show that gauge invariance is not affected by this prescription. Then by gauge invariance we are guaranteed that this will work for the longitudinal and pure gauge states also. Finally since gauge invariance is valid off-shell also, we can extrapolate to get the off-shell equations also.

To get the prescription for physical states let us consider the equation of motion for the state $Y_{n_{1}}^{\mu_{1}} Y_{n_{2}}^{\mu_{2}} \ldots Y_{n_{r}}^{\mu_{r}}$. The leading term is just

$$
\left(p^{2}+m^{2}\right) S_{n_{1} n_{2} . . n_{r}}^{\mu_{1} \mu_{2} \ldots \mu_{r}}=0
$$

where $m^{2}=n_{1}+n_{2}+\ldots+n_{r}-1$. Let us set $r=2$ for the moment. Consider the interaction between $S_{n_{1}}^{\mu_{1}}$ and $S_{n_{2}}^{\mu_{2}}$ to produce $S_{n_{1}, n_{2}}^{\mu_{1}}$. so that we have to look at the $a$-dependence in the integrated correlator of two vertex operators. The number of powers of $a$ from the vertex operators is $\left(n_{1}-1\right)+\left(n_{2}-1\right)$, the "classical" part and $p^{2}+q^{2}$, the anomalous part. If 
$z, w$ are the locations, then we have the integrals $\int d z \int d w$. Thus the final answer involves lookng at the coefficient of $\ln a$ in

$$
\int \frac{d z}{a} \int \frac{d w}{a}\left(\frac{z-w}{a}\right)^{2 p \cdot q} a^{p^{2}+q^{2}+2 p \cdot q} a^{n_{1}+n_{2}}
$$

We set all the particles on shell, $p^{2}+n_{1}-1=0$ and $q^{2}+n_{2}-1=0$. If we also set $(p+q)^{2}+n_{1}+n_{2}-1=0$ we see that all the powers of $a$ cancel except those coming from the regularization of the integration ( because $2 p . q \approx-1$ ) which gives a log divergence, whose coefficient is 1 . Alternatively if we keep all momenta slightly off-shell, the integration gives a result $\frac{1}{2 p \cdot q+1}$. When we do the operation $\frac{d}{d \ln a}$ we get $(p+q)^{2}+n_{1}+n_{2}-1$. When $p^{2}+n_{1}=1$ and $q^{2}+n_{2}=1$, the numerator zero cancels the pole and we get 1 . This gives the on-shell interaction between $S_{n_{1}}^{\mu_{1}}, S_{n_{2}}^{\mu_{2}}, S_{n_{1}, n_{2}}^{\mu_{1} \mu_{2}}$. If instead of including explicit factors of $a^{n_{1}}$ and $a^{n_{2}}$ with vertex operators, we naively let $p, q$ be 27 -dimensional then we will get $a^{\left(p^{26}+q^{26}\right)^{2}}$. This must provide the powers of $a$ that come from the vertex operators and the final undone integral which is $n_{1}+n_{2}-1$.

Thus we set $\left(p^{26}+q^{26}\right)^{2}=n_{1}+n_{2}-1$.

The only difference between the above calculation and the loop variable calculation is the expression $a^{(p+q)^{2}}$ becomes $e^{(p+q)^{2} \Sigma}$ in the loop variable calculation.

All this is easily generalized to the general case where $r>2$. We simply set $\left(\sum_{i=1}^{r} q_{0}\left(t_{i}\right)\right)^{2}=\sum_{i=1}^{r} n_{i}-1=P-1$. Thus our prescription is that in an expression $L_{n_{1}, n_{2}, \ldots, n_{r}}$ we must set $\left(\sum_{i=1}^{r} q_{0}\left(t_{i}\right)\right)^{2}=\sum_{i=1}^{r} n_{i}-1=P-1$. This can be achieved by setting all the $q_{0}\left(t_{i}\right)=\sqrt{\frac{P-1}{r}}$.

There is one other important point. Notice that in the term $(z-w)^{2 p . q}$ $p, q$ have to be 26 -dimensional momenta. So clearly the 27 th component does not modify the $z$-dependence of the correlation function. We will therefore set (letting $V$ denote the 26th direction) $K(z, w)^{V V}=\left\langle Y^{V}(z) Y^{V}(w)\right\rangle=$ $0 \forall z \neq w$. This effectively means setting $q_{n}(t,-z(t))=q_{n}(t, 0)$. This also affects the $\Sigma$ terms. We need not worry about the geometrical significance of what this means for the world sheet conformal theory of the 27th coordinate is concerned. The point is that gauge invariance is not affected because the transformation law (5.3.125) did not depend in any way on the $z$-dependence of the $k_{n}$. Thus to summarize: the prescription is to set $q_{0}=\sqrt{\frac{P-1}{r}}$, where $r$ is the number of fields in a term in the equation of motion. and $q_{n}(t,-z(t))=q_{n}(t, 0), \quad \forall n$.

Nor does this affect the consistency and uniqueness arguments of the 
previous section for defining gauge transformations of space-time fields. Provided we include the index $n$ of the gauge transformation parameter $\lambda_{n}$ in calculating $P$. This is because the gauge transformations are defined in terms of units that we denoted by $L_{i}$. Thus in a given $L_{i}, q_{0}$ has a well defined value which will determine the gauge transformation. For a different $L_{j}, q_{0}$ can take another value. If $L_{1}^{g}$ has terms of the same form as $L_{2}^{g}$ then they also necessarily have the same value of $P$ and hence of $q_{0}$ because the level must necessarily be the same. More precisely if $L_{1}^{g}+L_{2}^{g}=0$, this implies that $S_{1}^{g}+S_{2}^{g}=0$.

Thus at this point we have a set of equations that are correct when the particles are all on-shell. Furthermore they are gauge invariant on-shell and off-shell.

\subsection{Connection with RG}

We have already seen above that as far as on-shell physical modes are concerned the equation is obtained in exactly the same way as would be obtained had we done an RG operation $\frac{d}{d \ln a}$. However to make the formalism gauge invariant off shell also, we had to do a Taylor expansion of $Y(z)$. This means that to get the $z$-dependence (while keeping gauge invariance) we have to introduce the higher massive modes. Thus for instance if we want the equation involving $A^{\mu}$, which requires $k_{1}^{\mu}$, we must also use $\bar{k}_{2}^{\mu}(-z)$ because this contains in it $z k_{1}^{\mu}$. Once we use $\bar{k}_{2}^{\mu}(-z)$ we automatically have $k_{2}^{\nu}$. Thus massive modes are forced into the equation when we require gauge invariance off shell. This has been noticed earlier 41].

From the RG point of view this is natural. When we are off-shell we are away from the fixed point and one expects all the irrelevant operators to appear in the equation. These equations are of the form (2.1.7). The equations of motion for on-shell fields (marginal operators) is of the form (2.1.13), that gives the Callan-Symanzik (Gell-Mann - Low). $\beta$-function.

To see that the equations we get are indeed RG equations in the off-shell case let us count the powers of $a$ and show that $\frac{\delta}{\delta \Sigma}$ counts them. $\frac{\delta}{\delta \Sigma}$ brings down a factor of $\int d t_{1} \int d t_{2}\left(k_{0}^{\mu}\left(t_{1}\right) \cdot k_{0}^{\mu}\left(t_{2}\right)+q_{0}\left(t_{1}\right) \cdot q_{0}\left(t_{2}\right)\right)$, where $\mu: 0-25$. To make things simple we can work in dimensionless coordinates by replacing $z$ by $z / a$. The two point function $\left\langle X\left(\frac{z}{a}\right) X\left(\frac{w}{a}\right)\right\rangle=\frac{1}{2 \pi} \ln \left(1+\frac{(z-w)^{2}}{a^{2}}\right)$. In the original definition of the loop variable $Y_{n}$ came with a power of $a^{n}$. To compensate this we let $k_{n} \rightarrow k_{n} / a^{n}$. In this way it is clear that all the terms in $\bar{k}_{n}(-z / a)=\frac{k_{n}}{a^{n}}+\frac{z}{a} \frac{k_{n-1}}{a^{n-1}}+\ldots+k_{0} \frac{z^{n}}{a^{n}}$ all have the same dimension $n$. Thus $\int d t_{1} \int d t_{2} q_{0}\left(t_{1}\right) \cdot q_{0}\left(t_{2}\right)$, following our prescription above counts 
this dimension $n$. The anomalous $a$-dependence of $e^{i k \cdot X}$ which is $a^{k^{2}}$ and the overall $a$-dependence of $\left\langle: e^{i k \cdot X(z)}:: e^{i k \cdot X(w)}:\right\rangle=a^{2 p \cdot q}\left(1+\left(\frac{z-w}{a}\right)^{2}\right)^{2 p . q}$ which is $a^{2 p \cdot q}$ is what is counted by $\int d t_{1} \int d t_{2} k_{0}^{\mu}\left(t_{1}\right) \cdot k_{0}^{\mu}\left(t_{2}\right)$.

Thus we see that the term in the equation of motion which comes from $\int d t_{1} \int d t_{2}\left(k_{0}^{\mu}\left(t_{1}\right) \cdot k_{0}^{\mu}\left(t_{2}\right)+q_{0}\left(t_{1}\right) \cdot q_{0}\left(t_{2}\right)\right) \Sigma$ is the RG equation involving massive as well as massless modes. The terms coming from derivatives of $\Sigma$ are required for gauge invariance. Thus we see that we get a gauge invariant generalization of the of the exact Wilsonian RG equations by this procedure.

From the above discussion we see that the dimensionful coupling constant $k_{n}$ and $k_{0} z^{n}$ have the same dimension. Equivalently the dimensionless coupling can be defined by $k_{n}^{\prime}=\frac{k_{n}}{a^{n}}$. This is to be compare with $k_{0} \frac{z^{n}}{a^{n}}$. Now the upper limit in the range of $z$-integration, which can be denoted by $R$ is an infrared cutoff. After doing the integral we have to compare the irrelevant coupling $k_{n}$ with the marginal $k_{0}$ which occurs in the same equation as $k_{0} \frac{R^{n}}{a^{n}}$. Thus in the infrared limit $R \rightarrow \infty$ (or equivalently, in particle physics terminiology, the continuum limit $a \rightarrow 0$ ) the contribution of the irrelevant coupling is small, as expected. The ratio $\frac{R}{a}$ is an expansion parameter and measures the relative importance of the higher dimension operators corresponding to the irrelevant coupling constants. This is analogous to the number 4 in $(2.1 .7)$ or the level-expansion parameter $\frac{4}{3 \sqrt{3}}$ in BRST string field theory.

In [32] some of the higher order terms correcting Maxwell's equations were calculated.

The leading term in Maxwell's equation including the contribution of the tachyon is:

$\left(a^{2}\right)^{k_{0}^{2}}\left[\left(k^{2} i A^{\mu}(k)-A(k) . k i k^{\mu}\right)+(p+r)^{2} i A^{\mu}(p) \phi(r)-A(p) .(p+r) i(p+r)^{\mu} \phi(r)\right]$

A $z$-independent correction term involving massive modes:

$\left(a^{2}\right)^{k_{0}^{2}}\left(-\frac{4}{a^{2}}\right)\left\{(-i) \partial^{\mu} \partial^{\rho} \partial^{\sigma}\left[(1+\phi)\left(S_{2,1}^{\rho \sigma}+S_{2}^{\rho} A^{\sigma}\right)\right]+4 i \partial^{\mu}\left[i \partial^{\rho}\left[S_{3}^{\rho}(1+\phi)\right]+2 \sqrt{2} \partial^{\mu}\left(S_{3}(1+\phi)\right)\right.\right.$

We have set $\left(q_{0}\right)^{2}=2$.

A $z$-dependent term involving massless modes.

$$
\left(a^{2}\right)^{k_{0}^{2}}\left\{-6 \frac{z^{2}}{a^{2}} \partial_{\mu} \partial^{2} \partial^{\rho}\left[A^{\rho}(1+\phi)\right]+2 \frac{\left(z-z^{\prime}\right)^{2}}{a^{2}} \partial_{\mu} \partial^{\rho} \partial^{\sigma}\left[A^{\rho} \partial^{\sigma} \phi\right]\right\}
$$


We first rewrite $(5.6 .129)$ in terms of dimensionless coupling $S_{2,1}^{\prime}=\frac{S_{2,1}}{a^{2}}$. Comparing eqn. (5.6.129) with eqn. (5.6.130), we see that the contribution

of the marginal coupling $A$ is enhanced by the factor $\frac{R^{2}}{a^{2}}$ relative to $S_{2,1}^{\prime}$. Also comparing the corrections with the leading order term we see that an approximate solution will have $\alpha^{\prime} p^{2} A(p) \approx \frac{a^{2}}{R^{2}} A(p) \approx S_{2,1}^{\prime}$. This shows the relative contributions of relevant and irrelevant operators.

If we now naively take the limit $a \rightarrow 0$ we will be forced to set the e-m field to be a constant and all massive modes to zero. This will give us a "trivial" fixed point. If on the other hand if we keep $a$ finite and include all the higher correction terms with some critical values for the couplings, there is the possibility that they all add up to some closed form expression where the cutoff can be taken to zero. This would give a non-trivial fixed point.

A simple example of this is very familiar: The higher derivative terms involving $(z / a)^{n}$ and $\partial^{n} A$ (and products of $A$ ) are obviously expansions of terms of the form $\left(a^{2}+z^{2}\right)^{p . q}$ that are present in the Veneziano-type amplitude for scattering of photons. This is exactly what we get when we perform an operator product expansion of the the product of two vertex operators (but using the regularized Green's function). The product of two photon operators can be replaced by an infinite sum of massive mode vertex operators. Clearly when the photon is on-shell we can undo this expansion - then all these terms add up to some non-trivial but finite amplitude with a smooth limit $a \rightarrow 0$. The limit $a \rightarrow 0$ should only be taken after adding all these terms. One could also have more non-trivial examples such as the well known tachyon condensation [42].

\section{Conclusions}

In this paper we have dealt with the following question: How does one obtain gauge invariant equations of motion for all the modes of the open string using the renormalization group? Over and above the issue of gauge invariance there is the issue of going off shell. We have proposed a solution to this problem using loop variables. A condensed description of this proposal was given earlier in [32]. We have also tried to make clear the connection with the exact RG equation of Wilson and also the connection with the CallanSymanzik and Gell-Mann-Low equations. Zamolodchikov's c-theorem plays a role in this understanding. We have illustrated some of the ideas by applying them to known examples. 
Some intriguing features about this method were also mentioned in [32]. We mention it here also for completeness.

First, the theory is formally written as a massless theory in 27 dimensions and masses are obtained by a dimensional reduction prescription (that is quite a different one from the usual Kaluza-Klein reduction). Second, the structure of the interacting theory, both the form of the equations and the gauge transformation law, is similar to that of the free theory. The loop is just thickened to a band and the loop variables acquire a dependence on the positions of the vertex operators. Third, the gauge transformation law, in terms of loop variables has a simple interpretaion of space-time scale transformations. This supports the speculation [26] that the space-time Renormalization Group on a lattice with finite spacing, is part of the invariance group of string theory. Finally, space-time gauge invariance of the equations obtained this way does not seem tied down to any special world sheet properties, unlike in BRST string field theory where it follows from BRST invariance. To that extant it need not describe a string theory. Only the special choice of Green's function enforces the string theory connection. This may be a desirable feature from the point of view of the problem of background independence.

We also mention some of the open problems. We have not investigated the issue of whether there is a simple generalization that works for higher order string-loop corrections. It is also not known whether the equations follow from an action. The precise relation to BRST string field theory is not clear. The theory is so much simpler in terms of loop variables that it would be interesting to work out solution generating techniques in terms of these variables rather than in terms of space-time fields. Finally, it would be interesting to find a physical explanation of the "intriguing" features mentioned above.

\section{References}

[1] C. Lovelace, Phys. Lett. B135,75 (1984).

[2] C. Callan, D. Friedan, E. Martinec and M. Perry, Nucl. Phys. B262,593 (1985).

[3] A. Sen, Phys. Rev. D32,2102 (1985).

[4] E. Fradkin and A.A. Tseytlin, Phys. Lett. B151,316 (1985). 
[5] C. Callan and Z. Gan, Nucl. Phys. B272, 647 (1987)

[6] S. Das and B. Sathiapalan, Phys. Rev. Lett. B183,65 (1985).

[7] J. Hughes,J. Liu and J. Polchinski, Nucl. Phys. B316 (1989).

[8] T. Banks and E. Martinec, Nucl. Phys. B294, 733 (1987).

[9] V.A. Kostelecky, M.J. Perry, and R. Potting, Phys. Rev. Lett. 84 (2000) 4541, hepth/9912243.

[10] I.L. Buchbinder, O.M.Gitman,V.A. Krykhtin and Y.D. Pershin, hepth/9910188, Nucl. Phys. B584 (2000) 615.

[11] A.A. Tseytlin, Int. J. Mod. Phys. A4:4249 (1989) and references therein.

[12] K.G. Wilson and J. Kogut, Phys. Reports 12 , (1974)75.

[13] K.G.Wilson, Rev. Mod. Phys. 47, (1975)773.

[14] J. Polchinski, Nucl. Phys. B231 (1984) 269.

[15] K.G. Wilson, Phs. Rev. D7 (1973)2911.

[16] B. Sathiapalan, Nucl. Phys. B294, (1987) 747.

[17] A.B.Zamolodchikov, Sov. J. Nucl. Phys. 46(6) (1987)1090, Pis'ma Zh. Eksp. Teor. Fiz. 43 (1986) 565.

[18] A. Polyakov, "Gauge Fields and Strings", Harwood Academic Publishers, New York, 1987.

[19] E. Witten, hepth/9208027, Phys. Rev D 46 (1992) 5467; hepth/9210065, Phys. Rev. D 47 (9193) 3405.

[20] K. Li and E. Witten, hepth/9303067, Phys. Rev. D 48 (1993) 7297.

[21] S. Shatashvili, hepth/9303143, Phys. Lett B311 (1993)83; hepth/9311177.

[22] D. Kutasov, M. Marino, and G. Moore, hepth/0009148.

[23] E. Fradkin and A. A. Tseytlin, Phys. Lett. 163B, (1985) 123.

[24] A. Abouelsaood, C. G. Callan, C. R. Nappi and S. A. Yost, Nucl. Phys. B280,(1989) 599. 
[25] B. Sathiapalan, hepth/9509097; Intl. J. Mod. Phys. A 11 (1996) 2887.

[26] B. Sathiapalan, Nucl. Phys. B326 (1989)376.

[27] B. Sathiapalan, hepth/9512224; Mod. Phys. Lett. A11 (1996) 571.

[28] B. Sathiapalan, hepth/9704116, Talk given at the Third Puri Workshop, Dec 1996.

[29] B. Sathiapalan, Intl. J. Mod. Phys. A15 (2000)4761.

[30] B. Sathiapalan, Intl. J. Mod. Phys. A16 (2001) 1679.

[31] B. Sathiapalan, JHEP 0103 (2001)029.

[32] B. Sathiapalan, hepth/0201216; Mod. Phys. Lett. A17 (2002) 1175.

[33] B. Sathiapalan, hepth/9207051; Nucl. Phys. B405 (1993) 367.

[34] C. Fronsdal, Phys. Rev. D18 (1978) 3624.

[35] L. P. S. Singh and C. R. Hagen, Phys. Rev. D9 (1974) 898.

[36] W. Siegel and B. Zwiebach, Nucl. Phys. B263 (1986) 105.

[37] W. Siegel, Phys. Lett B149, 157; 162 (1984);B151 391;396 (1985).

[38] E. Witten, Nucl. Phys B268, (1986) 513.

[39] B. Sathiapalan, hepth/9511153;Mod. Phys. Lett. A11 (1996) 317.

[40] B. Sathiapalan, hepth/9207051; Nucl. Phys. B405, (1993) 367.

[41] B. Sathiapalan, hepth/9409023; Int. J. Mod. Phys. A10 (1995) 4501.

[42] A. Sen, hepth/9805170, JHEP 9808 (1998) 012.

[43] I. S. Gradshteyn and I. M. Ryzhik, Table of integrals, sines and products, (Academic Press, New York 1965). 